\title{
Synergistically Enhanced Mucoadhesive and Penetrable Polypeptide Nanogel for Efficient Drug Delivery to Orthotopic Bladder Cancer
}

\author{
Hui Guo, ${ }^{1,2}$ Faping Li, ${ }^{2}$ Heping Qiu $\mathbb{D}^{1,2}$ Weiguo $\mathrm{Xu}^{1,}{ }^{1}$ Pengqiang Li, ${ }^{1}$ Yuchuan Hou, ${ }^{2}$ \\ Jianxun Ding, ${ }^{1}$ and Xuesi Chen ${ }^{1}$ \\ ${ }^{1}$ Key Laboratory of Polymer Ecomaterials, Changchun Institute of Applied Chemistry, Chinese Academy of Sciences, \\ 5625 Renmin Street, Changchun 130022, China \\ ${ }^{2}$ Department of Urinary Surgery, The First Hospital of Jilin University, 71 Xinmin Street, Changchun 130021, China \\ Correspondence should be addressed to Jianxun Ding; jxding@ciac.ac.cn
}

Received 11 May 2019; Accepted 26 March 2020; Published 3 August 2020

Copyright (c) 2020 Hui Guo et al. Exclusive Licensee Science and Technology Review Publishing House. Distributed under a Creative Commons Attribution License (CC BY 4.0).

\begin{abstract}
Intravesical chemotherapy has been recommended after the gold standard of transurethral resection of the bladder tumor to prevent bladder cancer (BC) from local recurrence in the clinic. However, due to rapid urine excretion and barrier protection of the bladder wall, the clinical performances of chemotherapeutic drugs are severely compromised. In the present work, a smart positively charged disulfide-crosslinked nanogel of oligoarginine-poly(ethylene glycol)-poly(L-phenylalanine-co-L-cystine) $\left(\mathrm{R}_{9}\right.$-PEG-P(LP-co-LC)) was prepared to prolong the retention period and enhance the penetration capability of chemotherapeutic agent toward the bladder wall. PEG significantly improved the aqueous dispersibility of the 10-hydroxycamptothecin (HCPT)-loaded $\mathrm{R}_{9}$-PEG-P(LP-co-LC) (i.e., $\mathrm{R}_{9} \mathrm{NG} / \mathrm{HCPT}$ ) and enhanced the mucoadhesive capability by the nonspecific interaction between PEG chain and the bladder mucosa accompanied with the electrostatic interaction between the cationic $R_{9}$ and negatively charged bladder mucosa. Besides, $R_{9}$, as a cell-penetrating peptide, efficiently penetrated through the cell membrane and delivered carried cargo. The disulfide bond endowed the selective release behavior of HCPT triggered by the intracellular reductive microenvironment. As an advanced chemotherapeutic nanoformulation, the smart $\mathrm{R}_{9} \mathrm{NG} / \mathrm{HCPT}$ demonstrated superior cytotoxicity against human $\mathrm{BC} 5637$ cells in vitro and remarkably enhanced tumor suppression activity toward orthotopic BC models of mouse and rat in vivo, indicating its great potential in the clinical intravesical BC chemotherapy.
\end{abstract}

\section{Introduction}

Costs of curing bladder cancer (BC) are the highest on a perpatient basis because of the elevated rate of recurrence and multiple therapeutic interventions [1]. Over $75 \%$ of $\mathrm{BC}$ cases are initially diagnosed with non-muscle-invasive bladder cancer (NMIBC) [2]. The recommendation for NMIBC patients after transurethral resection of the bladder tumor is immediate intravesical chemotherapy, which has been proven to help prevent cancer from recurrence [3]. However, the clinical performances of chemotherapeutic drugs are severely compromised by rapid urine excretion and barrier protection of the bladder wall. Approximately $75 \%$ of patients will experience tumor relapse or progression within five years, which significantly affects their survival [4]. The five-year survival rate of BC patients has not been improved in the past three decades [5].
Nanomedicine, as revolutionary manufacturing technology, is expected to provide great opportunities to upregulate the exposure periods of drugs within the bladder. Currently, the leading materials, such as poly(ethylene glycol) (PEG) [6], chitosan [7], and liposomes [8], have been developed to improve the mucoadhesiveness or penetrability. PEG seems to be the most promising mucoadhesive polymer due to the nonspecific interaction between PEG chain and mucous membrane [9]. Moreover, PEG possesses the capability of improving the water solubility of hydrophobic drugs [10] and is well known for its reduced toxicity and low immunogenicity [11]. Cell-penetrating peptides (CPPs) are potentially less cytotoxic and possess the capacity of permeating through cell membrane to transport carried cargoes $[12,13]$. The combination of CPPs with anti-tumor drugs has been considered an incredibly attractive concept to improve 
therapeutic efficacy [14]. Among them, the arginine-rich CPPs have been demonstrated to be highly efficient in terms of cell uptake, especially the oligoarginine of nine arginine residues $\left(R_{9}\right)[15,16]$.

Accordingly, an innovative nanogel of $\mathrm{R}_{9}$-poly(ethylene glycol)-poly(L-phenylalanine-co-L-cystine) $\left(\mathrm{R}_{9}\right.$-PEG-P(LP-coLC)) was successfully synthesized to improve the mucoadhesiveness and penetrability of chemotherapeutical drugs (Scheme 1A). 10-Hydroxycamptothecin (HCPT) was applied as a model drug and encapsulated into the core of $\mathrm{R}_{9}-\mathrm{PEG}-$ $\mathrm{P}$ (LP-co-LC) by facile diffusion. The obtained drug-loaded nanogel was referred as $\mathrm{R}_{9} \mathrm{NG} / \mathrm{HCPT}$. The morphology of $\mathrm{R}_{9} \mathrm{NG} / \mathrm{HCPT}$ is similar to that of an octopus with spears in its hands, as shown in Scheme $1 \mathrm{~B}$. The rinsing of $\mathrm{R}_{9} \mathrm{NG} / \mathrm{HCPT}$ caused by urine excretion was resisted by the presence of PEG chains, which were like arms of an octopus, and $R_{9}$, which enhanced the mucoadhesion of $\mathrm{R}_{9} \mathrm{NG} / \mathrm{HCPT}$ through the nonspecific and electrostatic interactions, respectively. The powerful penetrating capacity of $\mathrm{R}_{9}$ overcame barrier protection of the bladder wall. $R_{9}$ was the weapon in the hands of octopus to break through obstacles. The drug-loaded core of polypeptide nanogel was much like the head of the octopus, which released the cargo triggered by the high concentration of glutathione (GSH) in BC cells [17].

The mucoadhesive nanogel (i.e., PLL-P(LP-co-LC)) reported previously was synthesized by the ring-opening polymerization (ROP) of $N(\varepsilon)$-benzyloxycarbonyl-L-lysine $N$-carboxyanhydride (ZLL NCA), L-phenylalanine $N$-carboxyanhydride (LP NCA), and L-cystine $N$-carboxyanhydride (LC NCA) [18]. The nanogel reported in this work, as distinct from PLL-P(LP-co-LC), introduced PEG and $\mathrm{R}_{9}$ and further magnified the mucoadhesion and penetration of $\mathrm{R}_{9} \mathrm{NG} / \mathrm{HCPT}$ as a benefit of the synergistic effect of PEG and $\mathrm{R}_{9}$.

The nanogel composed of PEG and polypeptide, but without $\mathrm{R}_{9}$, was synthesized as a negative control (i.e., NG/HCPT). In order to better understand the anti-tumor efficacy of $\mathrm{R}_{9} \mathrm{NG} / \mathrm{HCPT}$ in vivo, different orthotopic $\mathrm{BC}$ models of mouse and rat were constructed. The orthotopic BC model in mouse was constructed by instilling MB49 cells into the bladder that was pretreated with PLL solution [19]. The orthotopic BC model in rat was successfully established by intravesical instillation of $N$-methyl- $N$-nitrosourea (MNU). MNU played a vital role in the process of carcinogenesis, which was histologically indistinguishable from the human tumor [20]. The physicochemical properties, intracellular release profiles, and cytotoxicity of $\mathrm{R}_{9} \mathrm{NG} / \mathrm{HCPT}$ in vitro and mucoadhesiveness, permeability, biodistribution, antitumor activity, and systemic toxicity in vivo were systematically demonstrated. Encouragingly, $\mathrm{R}_{9} \mathrm{NG} / \mathrm{HCPT}$ possessed unique physicochemical properties to improve the chemotherapy efficacy of BC both in vitro and in vivo, as a potential benefit of strong urothelium adhesive property, high penetrating capacity, and selective drug release behavior.

\section{Results and Discussion}

2.1. Preparation and Characterizations of Empty and HCPTLoaded $R_{9}-P E G-P(L P-c o-L C)$. aPEG-P(LP-co-LC) was syn- thesized by the ROP of LP NCA and LC NCA with the amino-terminated allyloxy poly(ethylene glycol) (aPEG$\mathrm{NH}_{2}$ ) as a macroinitiator. $\mathrm{R}_{9}-\mathrm{PEG}-\mathrm{P}$ (LP-co-LC) was synthesized by the decoration of $R_{9}$ at the end of PEG by sequential deprotection, amidation, and Michael addition reactions, as depicted in Scheme 1A. The chemical structures of intermediates and the desired product $\mathrm{R}_{9}-\mathrm{PEG}-\mathrm{P}$ (LP-co-LC) were confirmed by the proton nuclear magnetic resonance $\left({ }^{1} \mathrm{H} \mathrm{NMR}\right)$ spectra. The characteristic resonance signals at $7.19 \mathrm{ppm}\left(\mathrm{e} ;-\mathrm{CH}_{2} \mathrm{C}_{6} \mathrm{H}_{5}, 5 \mathrm{H}\right)$ and $3.46 \mathrm{ppm}\left(\mathrm{d} ;-\mathrm{CH}_{2} \mathrm{C}_{6} \mathrm{H}_{5}\right.$, $2 \mathrm{H})$ were assigned to the protons in the L-phenylalanine unit. The peak at $2.42 \mathrm{ppm}\left(\mathrm{g} ;-\mathrm{SCH}_{2}-, 2 \mathrm{H}\right)$ was ascribed to protons of the L-cystine unit. The signal at $4.55 \mathrm{ppm}(\mathrm{c}+\mathrm{f}$; $\left.-\mathrm{COCH}\left(\mathrm{CH}_{2}\right) \mathrm{NH}-, 1 \mathrm{H}\right)$ was attributed to the protons of peptide backbone both in the polypeptide and $R_{9}$. The strong and broad signal peak of $b$ at $3.82 \mathrm{ppm}$ (b; $-\mathrm{OCH}_{2} \mathrm{CH}_{2}-, 4 \mathrm{H}$ ) was assigned to the methylene protons of PEG. The signal at $2.42 \mathrm{ppm}\left(\mathrm{a} ;-\mathrm{SCH}_{2} \mathrm{CH}_{2} \mathrm{CH}_{2}-, 2 \mathrm{H}\right)$ was produced by the thiol-ene click reaction between allyloxy and thiol groups. The peaks at 5.28 and $5.82 \mathrm{ppm}$ ( $\mathrm{a}$ and $\mathrm{b} ; \mathrm{CH}_{2}=\mathrm{CH}-, 2 \mathrm{H}$ and $1 \mathrm{H}$, respectively) were attributed to the allyloxy protons of aPEG-P(LP-co-LC), as shown in Supplementary Figure $\mathrm{S} 1$. The signal disappearance of the allyloxy protons (i) and the appearance of the $t$-butyloxycarbonyl ( $t$-Boc) resonance signal at $1.52 \mathrm{ppm} \quad\left(\mathrm{j} ; \quad\left(\mathrm{CH}_{3}\right)_{3} \mathrm{C}-, 9 \mathrm{H}\right)$ indicated the successful synthesis of $t$-Boc-NH-PEG-P(LP-co-LC), as depicted in Supplementary Figure S2 . The disappearance of $t$-Boc peak (j) demonstrated the successful synthesis of $\mathrm{NH}_{2}$-PEG$\mathrm{P}$ (LP-co-LC), as shown in Supplementary Figure S3. The maleimide-terminated polymer was produced by a simple amidation reaction, which was confirmed by the signal at $6.48 \mathrm{ppm}$ (h; $-\mathrm{CH}=\mathrm{CH}-, 2 \mathrm{H})$, as shown in Supplementary Figure S4B. The representative peaks at $1.4-4.2 \mathrm{ppm}$ in Supplementary Figure S4C matched precisely to the signals in Supplementary Figure S4A, and the signal disappearance of two vinyl protons on maleimide at $6.48 \mathrm{ppm}(\mathrm{h})$ both confirmed the successful synthesis of $\mathrm{R}_{9}-\mathrm{PEG}-\mathrm{P}$ (LP-co-LC). The nanogel of Mal-PEG-P(LP-co-LC) was synthesized as a negative control. The molar ratio of $\mathrm{LP}$ and $\mathrm{LC}$ in $\mathrm{R}_{9}-\mathrm{PEG}-$ $\mathrm{P}(\mathrm{LP}-\mathrm{co}$-LC) was calculated as 9:5 according to the content of carbon $(\mathrm{C})$, hydrogen $(\mathrm{H})$, nitrogen $(\mathrm{N})$, and sulfur $(\mathrm{S})$ determined by elemental analysis.

The drug loading contents (DLCs) of $\mathrm{R}_{9} \mathrm{NG} / \mathrm{HCPT}$ and NG/HCPT were up to $26.2 \pm 0.3$ and $25.6 \pm 0.4$ wt.\%, respectively. The drug loading efficiencies (DLEs) of $\mathrm{R}_{9} \mathrm{NG} / \mathrm{HCPT}$ and NG/HCPT were $88.9 \pm 1.4$ and $86.0 \pm 1.6 \mathrm{wt} . \%$, respectively. As depicted in Figure 1(a), the resulting $\mathrm{R}_{9} \mathrm{NG} / \mathrm{HCPT}$ displayed spherical morphology, which was detected by transmission electron microscopy (TEM). The hydrodynamic radius $\left(R_{\mathrm{h}}\right)$ of $\mathrm{R}_{9} \mathrm{NG} / \mathrm{HCPT}$ in phosphate-buffered saline (PBS) at $\mathrm{pH} 7.4$ was $48.9 \pm 0.7 \mathrm{~nm}$, while that of NG/HCPT was detected to be $44.8 \pm 1.3 \mathrm{~nm}$ (Figure 1(b)). Even when incubated with PBS for $48 \mathrm{~h}$, sizes of $\mathrm{R}_{9} \mathrm{NG} /$ HCPT and NG/HCPT almost maintained constant. However, after co-incubation with $10.0 \mathrm{mM}$ D,L-1,4-dithiothreitol (DTT) for $24 \mathrm{~h}$, the $R_{\mathrm{h}}$ of NG/HCPT increased to $401.0 \pm 50.3 \mathrm{~nm}$, and that of $\mathrm{R}_{9} \mathrm{NG} / \mathrm{HCPT}$ increased to $566.7 \pm 20.6 \mathrm{~nm}$, which could not be detected after $24 \mathrm{~h}$ 

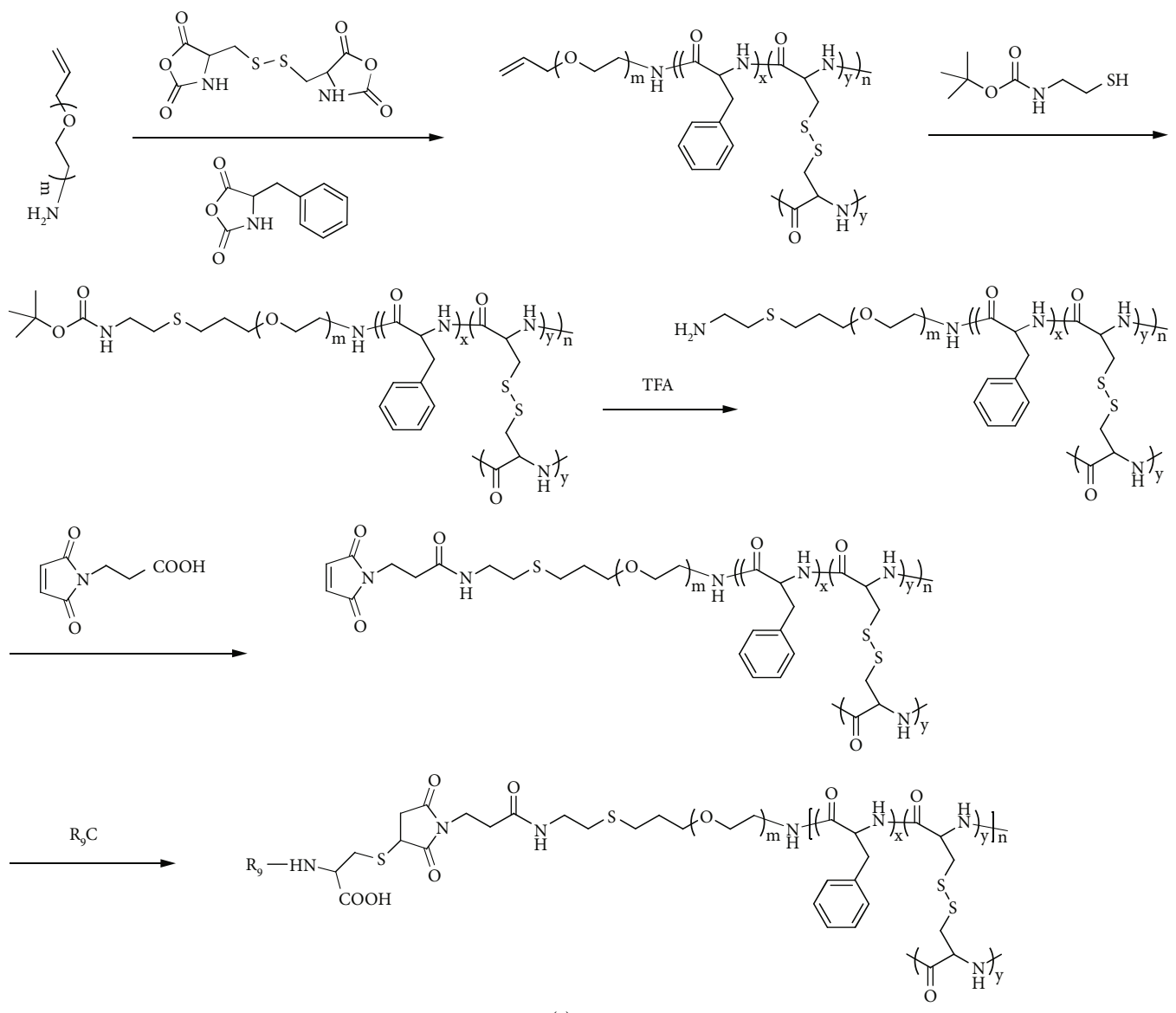

(a)

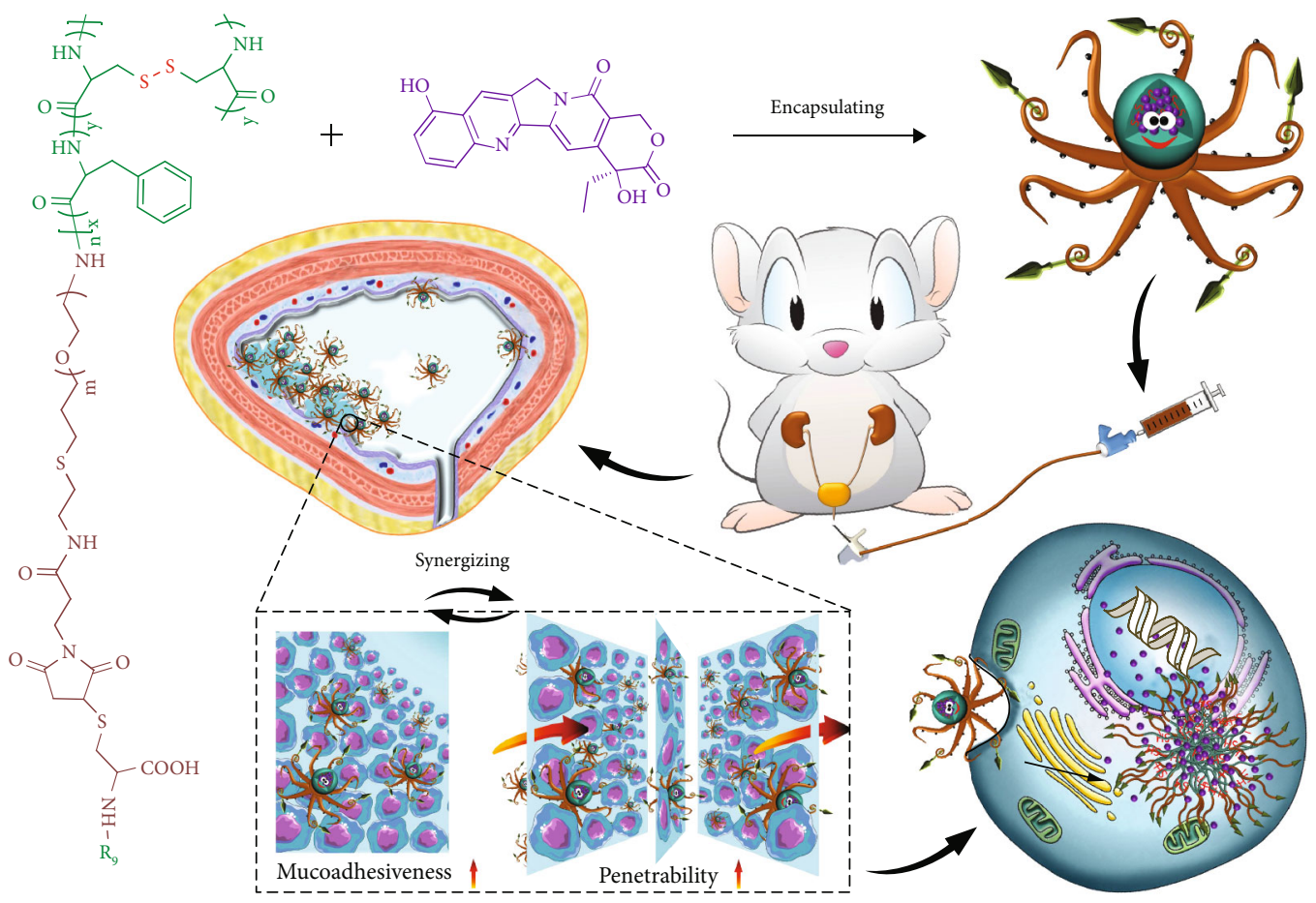

(b)

SCHEme 1: Synthesis and in vivo metabolism of water-soluble mucoadhesive nanogel. (A) Synthesis route of $\mathrm{R}_{9}-\mathrm{PEG}-\mathrm{P}(\mathrm{LP}-\mathrm{co}-\mathrm{LC})$. (B) Schematic illustration for self-assembly of $\mathrm{R}_{9} \mathrm{NG} / \mathrm{HCPT}$, intravesical chemotherapy of $\mathrm{R}_{9} \mathrm{NG} / \mathrm{HCPT}$, superior mucoadhesiveness, enhanced penetrability, selective accumulation in tumor tissue, promoted internalization, and accelerated $\mathrm{HCPT}$ release from $\mathrm{R}_{9} \mathrm{NG} / \mathrm{HCPT}$. 


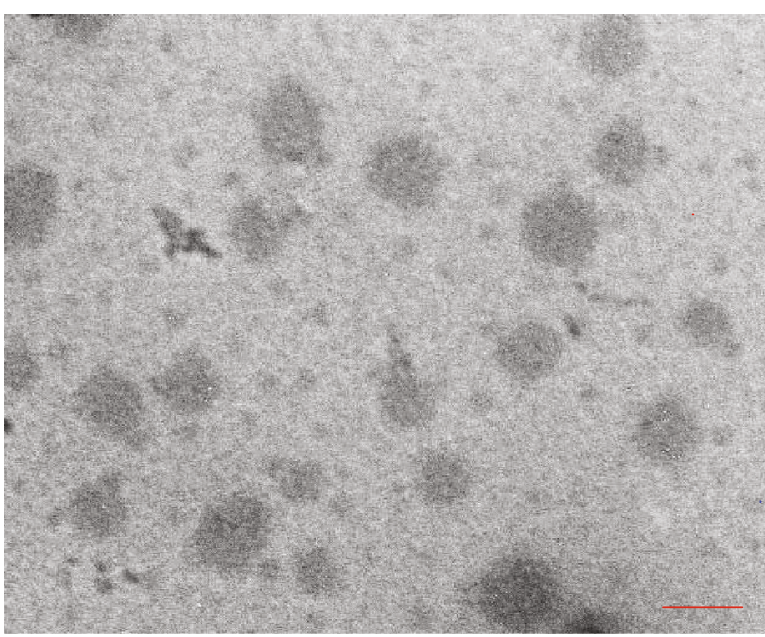

(a)

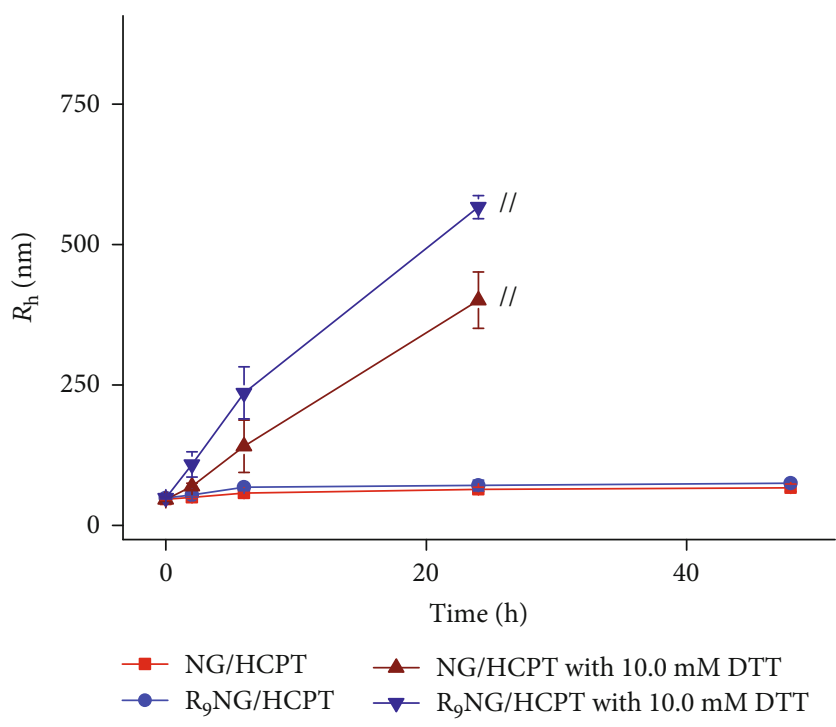

(c)

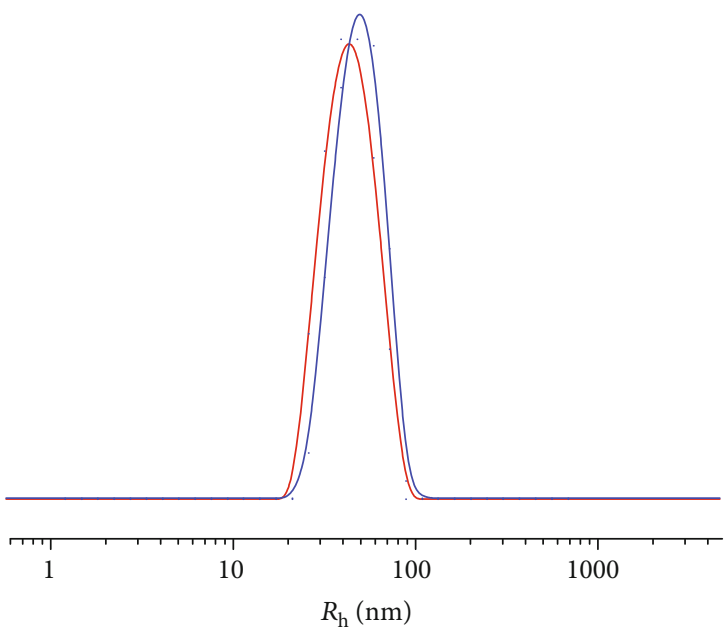

(b)

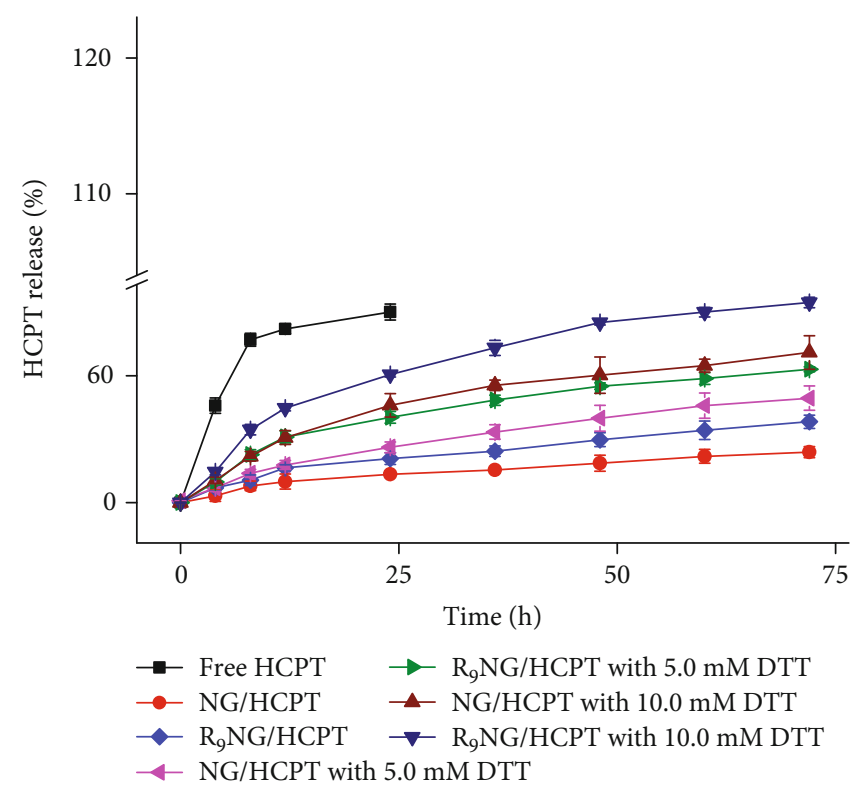

(d)

Figure 1: Characterizations of $\mathrm{R}_{9} \mathrm{NG} / \mathrm{HCPT}$. (a) Morphologies of $\mathrm{R}_{9} \mathrm{NG} / \mathrm{HCPT}$ in PBS by TEM. (b) $R_{\mathrm{h}}$ of $\mathrm{R}_{9} \mathrm{NG} / \mathrm{HCPT}$ in PBS. (c) Stability of $\mathrm{R}_{9} \mathrm{NG} / \mathrm{HCPT}$ in PBS without or with $10.0 \mathrm{mM}$ DTT versus time. The scale bar in (a) represents $100 \mathrm{~nm}$. // represents the interruption of $R_{\mathrm{h}}$-related light-scattering signal. (d) In vitro release profiles of $\mathrm{R}_{9} \mathrm{NG} / \mathrm{HCPT}$. All the statistical data are represented as mean \pm standard deviation $(n=3)$.

(Figure 1(c)). The results demonstrated the reductionresponsive properties of NG/HCPT and $\mathrm{R}_{9} \mathrm{NG} / \mathrm{HCPT}$, which were critical for the selective delivery of drugs to cancer cells.

The zeta potential of NG/HCPT was $-17.3 \pm 0.8 \mathrm{mV}$, while that of $\mathrm{R}_{9} \mathrm{NG} / \mathrm{HCPT}$ was $26.9 \pm 1.2 \mathrm{mV}$. The positive charge of $\mathrm{R}_{9} \mathrm{NG} / \mathrm{HCPT}$ further demonstrated the successful conjugation of $\mathrm{R}_{9}$ onto the surface of the nanogel, which could enhance the interaction between $\mathrm{R}_{9} \mathrm{NG} / \mathrm{HCPT}$ and negatively charged cell membrane and subsequently upregulated cell internalization [21]. The release behavior of HCPT from $\mathrm{R}_{9} \mathrm{NG} / \mathrm{HCPT}$ was examined in $\mathrm{PBS}$ at $\mathrm{pH} 7.4$ with $0.1 \%(W / V)$ Tween-80 containing different concentrations of DTT. As shown in Figure 1(d), free HCPT displayed a rapid diffusion from the dialysis membrane within $12 \mathrm{~h}$. In contrast, $\mathrm{R}_{9} \mathrm{NG} / \mathrm{HCPT}$ released only $38.2 \%$ of HCPT throughout $72 \mathrm{~h}$ in PBS without DTT, demonstrating excellent stability of $\mathrm{R}_{9} \mathrm{NG} / \mathrm{HCPT}$ under physiological conditions. In addition, the release rate of $\mathrm{HCPT}$ from $\mathrm{R}_{9} \mathrm{NG} / \mathrm{HCPT}$ was remarkably accelerated due to the increased concentration of DTT. The cumulative release amount of HCPT was $62.9 \%$ and $94.5 \%$ over a period of $72 \mathrm{~h}$ with the concentration of DTT ranging from 5.0 to $10.0 \mathrm{mM}$, respectively. The results demonstrated the excellent physiological stability of $\mathrm{R}_{9} \mathrm{NG} / \mathrm{HCPT}$. However, the disulfide bond could be rapidly broken in the reductive microenvironment, resulting in the selective HCPT release in the cancer cells. 
2.2. Intracellular Release and Cytotoxicity of $R_{9} N G / H C P T$. The intracellular release behavior of $\mathrm{R}_{9} \mathrm{NG} / \mathrm{HCPT}$ was qualitatively evaluated by confocal laser scanning microscopy (CLSM). Figure 2(a) presents the CLSM images of human BC 5637 cells after co-incubation with free HCPT, NG/HCPT, or $\mathrm{R}_{9} \mathrm{NG} / \mathrm{HCPT}$ with equivalent HCPT concentration at $1.25 \mu \mathrm{g} \mathrm{mL}^{-1}$. The cells depicted slightly weaker HCPT fluorescence after co-incubation with $\mathrm{R}_{9} \mathrm{NG} / \mathrm{HCPT}$ for $2 \mathrm{~h}$ as compared with that of free HCPT. At the same point, the fluorescence intensity of negatively charged NG/HCPT was the weakest. Although it was not as simple as small-molecule drugs entering cells by diffusion, the results demonstrated that $\mathrm{R}_{9} \mathrm{NG} / \mathrm{HCPT}$ could be internalized by human BC 5637 cells effectively [22]. Furthermore, after the co-incubation period increased up to $6 \mathrm{~h}$, the cells treated with $\mathrm{R}_{9} \mathrm{NG} / \mathrm{HCPT}$ exhibited more robust $\mathrm{HCPT}$ fluorescence than that of free HCPT or NG/HCPT (Figure 2(a)). The significantly enhanced HCPT fluorescence mainly benefited from the electrostatic interaction between the positively charged $\mathrm{R}_{9} \mathrm{NG} / \mathrm{HCPT}$ and the negatively charged cell membrane [23]. The conjugated $\mathrm{R}_{9}$ facilitated the cell uptake of $\mathrm{R}_{9} \mathrm{NG} / \mathrm{HCPT}$ through the CPP-mediated cell entry [24]. In addition, the high concentration of GSH in human BC 5637 cells led to the rupture of the disulfide bond and accelerated the HCPT release from $\mathrm{R}_{9} \mathrm{NG} / \mathrm{HCPT}$ [25].

The cell uptake and subsequent release profiles were investigated quantitatively according to the protocol described by Wei et al. [26]. Compared with free HCPT, the cells cocultured with $\mathrm{R}_{9} \mathrm{NG} / \mathrm{HCPT}$ displayed a slightly lower content of HCPT at $2 \mathrm{~h}$. The accumulation of HCPT in cells cocultured with NG/HCPT was the lowest at the same time point. With the prolongation of co-incubation time, the endocytosis of $\mathrm{R}_{9} \mathrm{NG} / \mathrm{HCPT}$ increased significantly, and a relatively higher content of $\mathrm{HCPT}$ was maintained as compared to that of free HCPT or NG/HCPT from the second time point. Conversely, the increased HCPT concentration in the cells of the $\mathrm{R}_{9} \mathrm{NG} / \mathrm{HCPT}$ group was about 1.9 times compared with that in the cells treated with free HCPT (Figure 2(b)), while it was about 1.5 times as much as that in the NG/HCPT group at $6 \mathrm{~h}$. The results were consistent with the representative finding by CLSM (Figure 2(a)).

To evaluate the potential cytotoxicity of $\mathrm{R}_{9} \mathrm{NG} / \mathrm{HCPT}$ against human BC 5637 cells, a standard methyl thiazolyl tetrazolium (MTT) assay was performed. The cytotoxicity of free HCPT, NG/HCPT, and $\mathrm{R}_{9} \mathrm{NG} / \mathrm{HCPT}$ was enhanced in a concentration-dependent manner (Figure 2(c)). Furthermore, the cytotoxicity of $\mathrm{R}_{9} \mathrm{NG} / \mathrm{HCPT}$ surpassed those of free HCPT and NG/HCPT against human BC 5637 cells. The superior cytotoxic effect of $\mathrm{R}_{9} \mathrm{NG} / \mathrm{HCPT}$ probably benefited from the weak positive surface, $R_{9}$, and the reduction-responsive property of $\mathrm{R}_{9} \mathrm{NG} / \mathrm{HCPT}$, which led to the promoted cell uptake and accelerated intracellular HCPT release in human BC 5637 cells. Importantly, the half-maximal inhibitory concentration $\left(\mathrm{IC}_{50}\right)$ of $\mathrm{R}_{9} \mathrm{NG} / \mathrm{HCPT}$ was $2.4 \pm 0.1 \mu \mathrm{g} \mathrm{m}^{-1}$, which was lower in comparison with those of free HCPT (i.e., $8.7 \pm 0.8 \mu \mathrm{g} \mathrm{m}^{-1}$ ) and $\mathrm{NG} / \mathrm{HCPT}$ (i.e., $4.7 \pm 0.2$ $\left.\mu \mathrm{g} \mathrm{mL}^{-1}\right)$. The lowest $\mathrm{IC}_{50}$ indicated the potent cytotoxicity of $\mathrm{R}_{9} \mathrm{NG} / \mathrm{HCPT}$ quantitatively, suggesting the superiority of $\mathrm{R}_{9} \mathrm{NG} / \mathrm{HCPT}$ for intravesical chemotherapy of $\mathrm{BC}$ in vivo.
The $\mathrm{R}_{9} \mathrm{NG} / \mathrm{HCPT}$-induced apoptosis of human BC 5637 cells was measured by flow cytometry (FCM) analysis. As shown in Figure 2(d), the $24 \mathrm{~h}$ exposure to three different formulations of HCPT at an equivalent HCPT dosage of $0.1 \mu \mathrm{g} \mathrm{mL}^{-1}$ resulted in a noticeable difference in the percentage of apoptotic cells. $\mathrm{R}_{9} \mathrm{NG} / \mathrm{HCPT}$ contributed most to an abundance of apoptotic cells. The total percentage of apoptotic cells was $22.5 \%$, which was constituted with $16.5 \%$ early apoptotic cells and $6.0 \%$ late apoptotic cells. In contrast, the total percentage of cells displaying apoptosis was reduced to $12.2 \%$ and $18.7 \%$ after co-incubation with free HCPT and NG/HCPT, respectively. The results were consistent with the cytotoxicity of $\mathrm{R}_{9} \mathrm{NG} / \mathrm{HCPT}$ described above. When the co-incubation time was prolonged to $48 \mathrm{~h}$, the differences among free HCPT, NG/HCPT, and $\mathrm{R}_{9} \mathrm{NG} / \mathrm{HCPT}$ groups varied greatly, as shown in Supplementary Figure S5. The total percentage of cells displaying apoptosis was increased to $38.9 \%, 53.5 \%$, or $63.0 \%$ after co-incubation with free HCPT, NG/HCPT, or $\mathrm{R}_{9} \mathrm{NG} / \mathrm{HCPT}$, respectively. This phenomenon indicated that different formulations induced the apoptosis of human BC 5637 cells in a time-dependent manner. The increased apoptotic activity induced by $\mathrm{R}_{9} \mathrm{NG} / \mathrm{HCPT}$ benefited from the high intracellular concentration of HCPT, which was internalized through CPP-mediated endocytosis and triggered to release by GSH rapidly.

2.3. Mucoadhesiveness, Permeability, and Biodistribution of $R_{9} N G / H C P T$. CLSM was employed to demonstrate the mucoadhesiveness and permeability of $\mathrm{R}_{9} \mathrm{NG} / \mathrm{HCPT}$. The male Sprague-Dawley (SD) rats with $\mathrm{BC}$ were anesthetized and then administrated with different HCPT formulations at a consistent HCPT dosage of $6.0 \mathrm{mg}$ per kg body weight $\left(\mathrm{mg}(\mathrm{kg} \mathrm{BW})^{-1}\right)$ through bladder irrigation. At different indicated time points (i.e., $0.5,2,6,12,24$, and $48 \mathrm{~h}$ ), the bladders were removed and sectioned.

As depicted in Figure 3(a), the fluorescence signals of free $\mathrm{HCPT}$ and $\mathrm{R}_{9} \mathrm{NG} / \mathrm{HCPT}$ were observed in the bladder sections at the first few time points. The fluorescence intensity of $\mathrm{R}_{9} \mathrm{NG} / \mathrm{HCPT}$ was stronger than that of free HCPT. The highest fluorescence signal was observed in the bladder treated with $\mathrm{R}_{9} \mathrm{NG} / \mathrm{HCPT}$ at $0.5 \mathrm{~h}$. Not surprisingly, the fluorescence intensity of $\mathrm{R}_{9} \mathrm{NG} / \mathrm{HCPT}$ decreased over time. However, it remained at a relatively high concentration even at $12 \mathrm{~h}$, indicating the high mucoadhesion of $\mathrm{R}_{9} \mathrm{NG} /$ HCPT toward the urothelial surface. On the contrary, the fluorescence signal in the bladder exposed to free HCPT decreased rapidly, which was attributed to the continuous excretion of urine. This phenomenon demonstrated the outstanding mucoadhesiveness of $\mathrm{R}_{9} \mathrm{NG} / \mathrm{HCPT}$. In order to quantitatively prove the mucoadhesiveness of $\mathrm{R}_{9} \mathrm{NG} / \mathrm{HCPT}$, the average optical density of HCPT was measured. As verified in Figure $3(\mathrm{~b})$, the fluorescence intensity of $\mathrm{R}_{9} \mathrm{NG} / \mathrm{HCPT}$ was 1.6 times higher as compared with that of free HCPT at $0.5 \mathrm{~h}$. Inspiringly, the fluorescence intensity increased from 2.2 to 4.8 times when the retention time was prolonged from 2 to $6 \mathrm{~h}$. The outstanding mucoadhesiveness of $\mathrm{R}_{9} \mathrm{NG} / \mathrm{HCPT}$ benefited from the nonspecific interaction between PEG chain and the bladder mucosa and electrostatic interaction between terminal cationic $R_{9}$ and negatively charged surface 


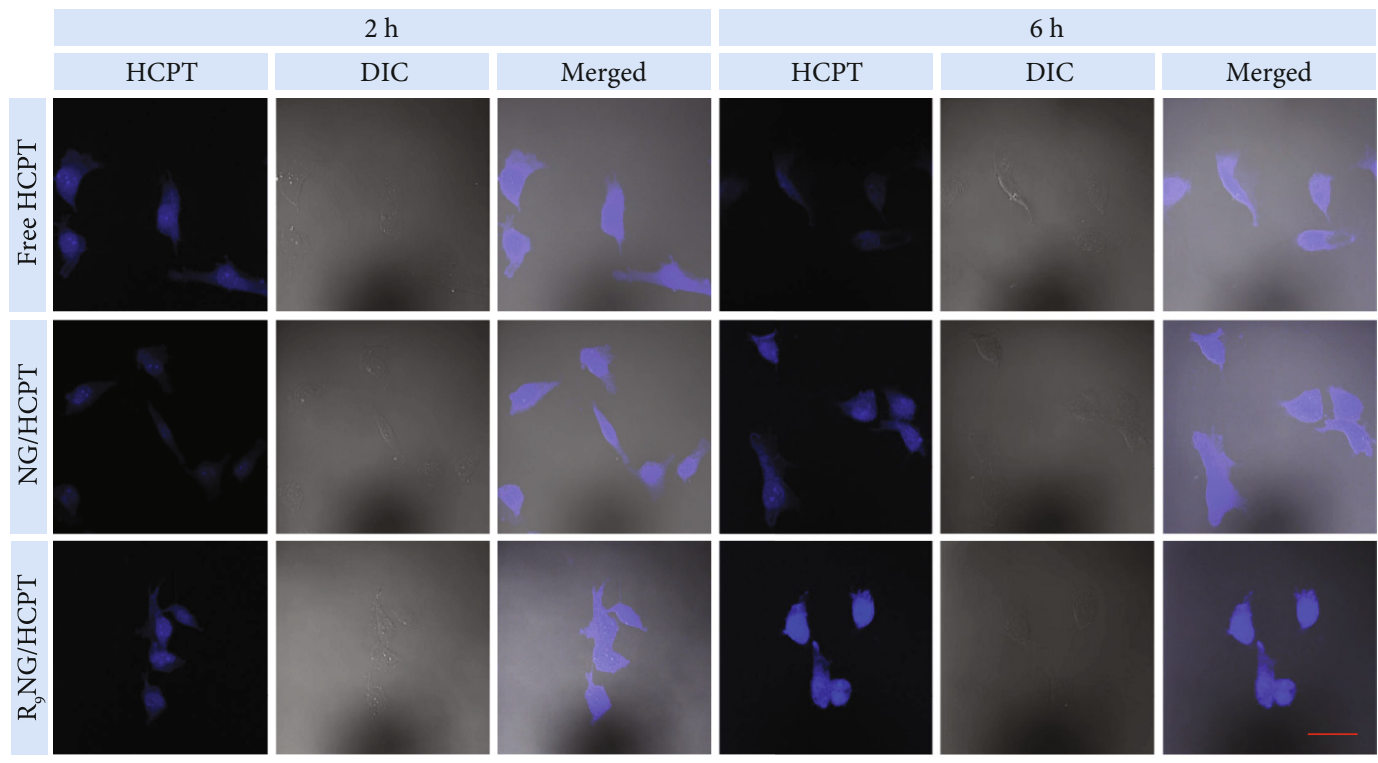

(a)

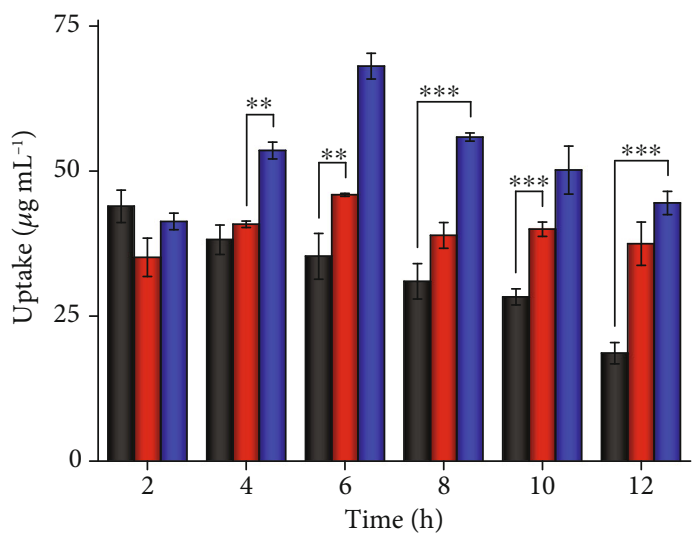

Free HCPT

NG/HCPT

$\mathrm{R}_{9} \mathrm{NG} / \mathrm{HCPT}$

(b)

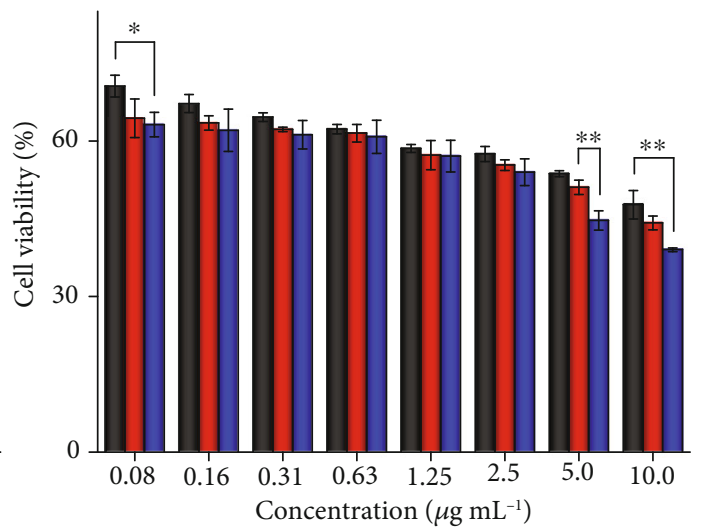

Free HCPT

$\square$ NG/HCPT

$\square \mathrm{R}_{9} \mathrm{NG} / \mathrm{HCPT}$
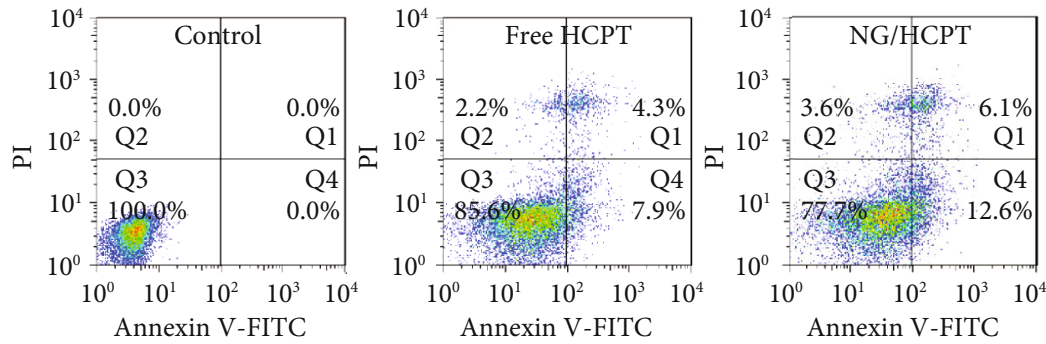

(c)

(d)

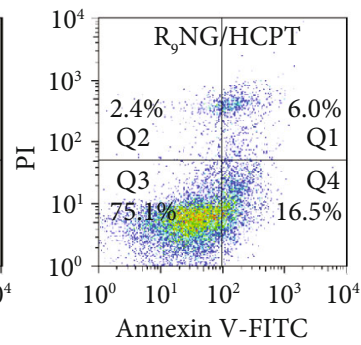

Figure 2: In vitro cell uptake and cytotoxicity. (a) Representative CLSM images of human BC 5637 cells incubated with free HCPT, NG/HCPT, or $\mathrm{R}_{9} \mathrm{NG} / \mathrm{HCPT}$ for 2 or $6 \mathrm{~h}$. The blue color was HCPT fluorescence in cells (left). Differential interference contrast (DIC) was used to obtain the bright-field images (middle), and the merged images were shown on the right. The scale bar represents $50 \mu \mathrm{m}$. (b) Intracellular HCPT release profiles in human BC 5637 cells after co-incubation with free HCPT, NG/HCPT, or R ${ }_{9} \mathrm{NG} / \mathrm{HCPT}$. Data are presented as mean \pm standard deviation $\left(n=3 ;{ }^{*} P<0.05,{ }^{* *} P<0.01\right.$, and $\left.{ }^{* * *} P<0.001\right)$. (c) The viability of human BC 5637 cells incubated with free HCPT, NG/HCPT, or $\mathrm{R}_{9} \mathrm{NG} / \mathrm{HCPT}$ at different concentrations for $24 \mathrm{~h}$. Data are presented as mean \pm standard deviation $\left(n=3 ;{ }^{*} P<0.05,{ }^{* *} P<0.01\right)$. (d) Apoptotic cell populations were calculated by FCM analysis after co-incubating human BC 5637 cells with PBS as a control, free HCPT, NG/HCPT, or $\mathrm{R}_{9} \mathrm{NG} / \mathrm{HCPT}$ for $24 \mathrm{~h}$. The lower-left (Q3), lower-right (Q4), upper-right (Q1), and upper-left (Q2) quadrants in each panel indicated the populations of healthy, early and late apoptotic, and necrotic cells, respectively. 

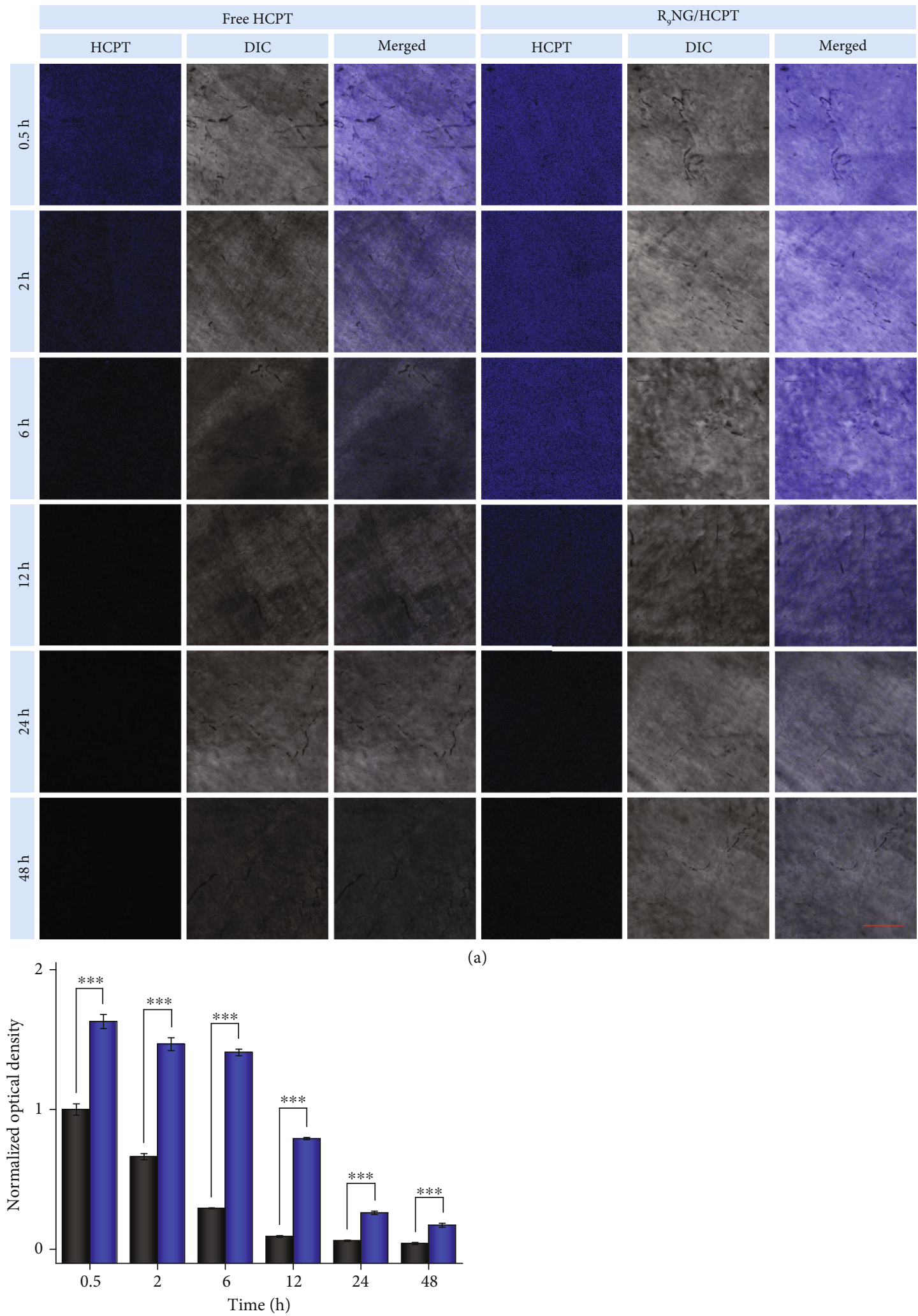

(a)

Free HCPT

$\mathrm{R}_{9} \mathrm{NG} / \mathrm{HCPT}$

(b)

Figure 3: Mucoadhesiveness. (a) The fluorescence intensities of free HCPT and $\mathrm{R}_{9} \mathrm{NG/HCPT}$ adhered to the inner surface of the bladders were determined by CLSM. (b) Quantitative evaluation of HCPT fluorescence intensities from results by CLSM. The scale bar in (a) represents $100 \mu \mathrm{m}$. Data are presented as mean \pm standard deviation $\left(n=3 ;{ }^{* * *} P<0.001\right)$. 
of the bladder mucosa, which retarded the wash-off of $\mathrm{R}_{9} \mathrm{NG} / \mathrm{HCPT}$ by urine $[27,28]$.

The permeability of $\mathrm{R}_{9} \mathrm{NG} / \mathrm{HCPT}$ was verified in Figure 4(a). Within half an hour, a much stronger fluorescence signal was observed in the full-thickness bladder wall treated with free HCPT and especially confined to the mucous membrane. At the same time, $\mathrm{R}_{9} \mathrm{NG} / \mathrm{HCPT}$ displayed a shallow penetration within the bladder wall. However, $\mathrm{R}_{9} \mathrm{NG} / \mathrm{HCPT}$ gradually penetrated through the full-thickness bladder over time. It also maintained a relatively stronger HCPT fluorescence than free HCPT for an extended period. The results indicated the exceptional permeability and accumulation of $\mathrm{R}_{9} \mathrm{NG} / \mathrm{HCPT}$ in the bladder. For detailed analysis of the permeability of $\mathrm{R}_{9} \mathrm{NG} / \mathrm{HCPT}$, the HCPT fluorescence signals were normalized to the maximum value. As shown in Figure 4(b), the fluorescence intensity of $\mathrm{R}_{9} \mathrm{NG} / \mathrm{HCPT}$ gradually spread throughout the whole bladder tissue over time and remained a comparatively higher HCPT content. The phenomenon was mainly due to the strong penetration ability of $R_{9}$ with a positive surface charge $[29,30]$. In addition, the advanced mucoadhesiveness of $\mathrm{R}_{9} \mathrm{NG} / \mathrm{HCPT}$ further promoted its permeability. An excellent chemotherapeutic effect of $\mathrm{R}_{9} \mathrm{NG} / \mathrm{HCPT}$ was predictable based on superior permeability, which was vital for the treatment of $\mathrm{BC}$.

The bladder accumulation of $\mathrm{R}_{9} \mathrm{NG} / \mathrm{HCPT}$ was verified by high-performance liquid chromatography (HPLC). Male SD rats were administrated with intravesical instillation of different HCPT solutions, and the experimental rats were euthanized at $6 \mathrm{~h}$. Subsequently, the bladders and other major organs, such as the heart, liver, spleen, lung, and kidney, were excised and homogenized. Then, the samples were extracted with the organic phase of methanol and acetonitrile $(1 / 1, V / V) .20 .0 \mu \mathrm{L}$ of mixture suspension collected by centrifugation was analyzed for the determination of drug content. HCPT, released from $\mathrm{R}_{9} \mathrm{NG} / \mathrm{HCPT}$, was mainly accumulated in the bladder, which was 2.2 times higher as compared with free HCPT (Figure S6, Supporting Information). In addition, the other organs provided extremely low drug content. The results confirmed the advanced mucoadhesiveness of $\mathrm{R}_{9} \mathrm{NG} /$

HCPT and predicted an improved anti-tumor efficacy of $\mathrm{R}_{9} \mathrm{NG} / \mathrm{HCPT}$ with reduced toxicity on normal organs.

2.4. In Vivo Anti-Tumor Efficacy of $R_{9} N G / H C P T$. The anti-tumor efficacy of $\mathrm{R}_{9} \mathrm{NG} / \mathrm{HCPT}$ was assessed on the orthotopic BC in C57Bl/6 mice. Briefly, PLL $(100.0 \mu \mathrm{L}$ of $0.1 \mathrm{mg} \mathrm{mL}^{-1}$ for $20 \mathrm{~min}$ ) was used to disrupt the glycosaminoglycan layer. $50.0 \mu \mathrm{L}$ of MB49 cell suspension, including $1.0 \times 10^{5}$ cells, was slowly injected into the bladder of C57Bl/6 mouse, and the cells were allowed to dwell in the bladder for $50 \mathrm{~min}$ [31]. The tumor-bearing mice were randomly divided into four groups: PBS as a control, free HCPT, NG/HCPT, and $\mathrm{R}_{9} \mathrm{NG} / \mathrm{HCPT}(n=6)$. Different HCPT formulations were administered weekly by catheterization at a dosage of $6.0 \mathrm{mg}(\mathrm{kg} \mathrm{BW})^{-1}$ for a total of four treatments. To define the development of $\mathrm{BC}$, especially the tumor size, cystography was performed. Polypoid filling defects and irregular surface were manifested in cystography after treat- ment, which was depicted in Figure 5(a). The most extensive space-occupying lesion was identified in the bladder exposed to PBS, while there was no significant abnormality recognized in the bladder administrated with $\mathrm{R}_{9} \mathrm{NG} / \mathrm{HCPT}$ based on the smooth margin. The superior anti-tumor efficacy of $\mathrm{R}_{9} \mathrm{NG} / \mathrm{HCPT}$ was intuitionally approved by cystography. The result was mostly attributed to the presence of PEG and $R_{9}$ in $R_{9} N G / H C P T$, which could eventually result in a remarkably enhanced mucoadhesiveness and permeability to improve the anti-tumor efficacy synergistically. The suboptimal BC growth inhibition of free HCPT was caused by rapid drug excretion from urine.

The anti-tumor efficacy of $\mathrm{R}_{9} \mathrm{NG} / \mathrm{HCPT}$ was further assessed on another orthotopic $\mathrm{BC}$ in male SD rats. The orthotopic BC was established by four doses of MNU [32]. Histopathological analysis was performed to demonstrate the successful induction of BC after the intravesical instillation of MNU for eight weeks. As verified in Supplementary Figure S7A, the abundance of cancer cells was observed with apparent architectural disarray and nuclear atypia, indicating the occurrence of $\mathrm{BC}$. The $\mathrm{BC}$-bearing rats were randomly divided into three groups: $\mathrm{PBS}$ as a control, free $\mathrm{HCPT}$, and $\mathrm{R}_{9} \mathrm{NG} / \mathrm{HCPT}(n=8)$. The equivalent HCPT dosage of $6.0 \mathrm{mg}(\mathrm{kg} \mathrm{BW})^{-1}$ was given by intravesical instillation weekly for a total of six treatments. Cystography was performed to define the development of BC. As pointed in Supplementary Figure S7B, the results were consistent with those of cystography in mice (Figure 5(a)).

The bladders were harvested at the end of the treatments to assess the external surface, which was shown in Figure 5(b) and Figure S7B, Supporting Information. A rigid-walled bladder with the largest volume was detected in the PBS group, suggesting that exuberant growth of the tumor had infiltrated the muscular layer of the bladder wall. In contrast, a smooth-surfaced bladder with standard size and general shape occurred in the $\mathrm{R}_{9} \mathrm{NG} / \mathrm{HCPT}$ group. The bladders exposed to free HCPT or NG/HCPT were generally less rigid, suggesting that free HCPT and NG/ HCPT were effective but less effective than $\mathrm{R}_{9} \mathrm{NG} / \mathrm{HCPT}$. To be better informed of the growth of the tumor, the bladders were opened up, and the internal surface was displayed (Figure 5(b) and Figure S7B, Supporting Information). Multiple neoplasms with a "fish flesh" soft tan appearance were found in the bladder cavity of the control group, while there was no obvious abnormality in the bladder treated with $\mathrm{R}_{9} \mathrm{NG} / \mathrm{HCPT}$. The bladder observation intuitively confirmed that $\mathrm{R}_{9} \mathrm{NG} / \mathrm{HCPT}$ possessed an excellent anti-tumor efficacy on $\mathrm{BC}$ both in mouse and rat.

In order to evaluate the systemic toxicity of $\mathrm{R}_{9} \mathrm{NG} / \mathrm{HCPT}$, the body weights of the experimental animals were monitored once a week and shown in Figure 5(c) and Figure S7C, Supporting Information. The experimental animals ad-ministrated with $\mathrm{R}_{9} \mathrm{NG} / \mathrm{HCPT}$ showed a stable increase in body weight during the treatment, indicating negligible systemic toxicity of $\mathrm{R}_{9} \mathrm{NG} / \mathrm{HCPT}$. Another essential pre-dictor of efficacies and toxicity of various drug formulations was the survival rate, which was shown in Figure 5(d) and Figure S7D, Supporting Information [33]. Different HCPT formulations extended the overall survivals 


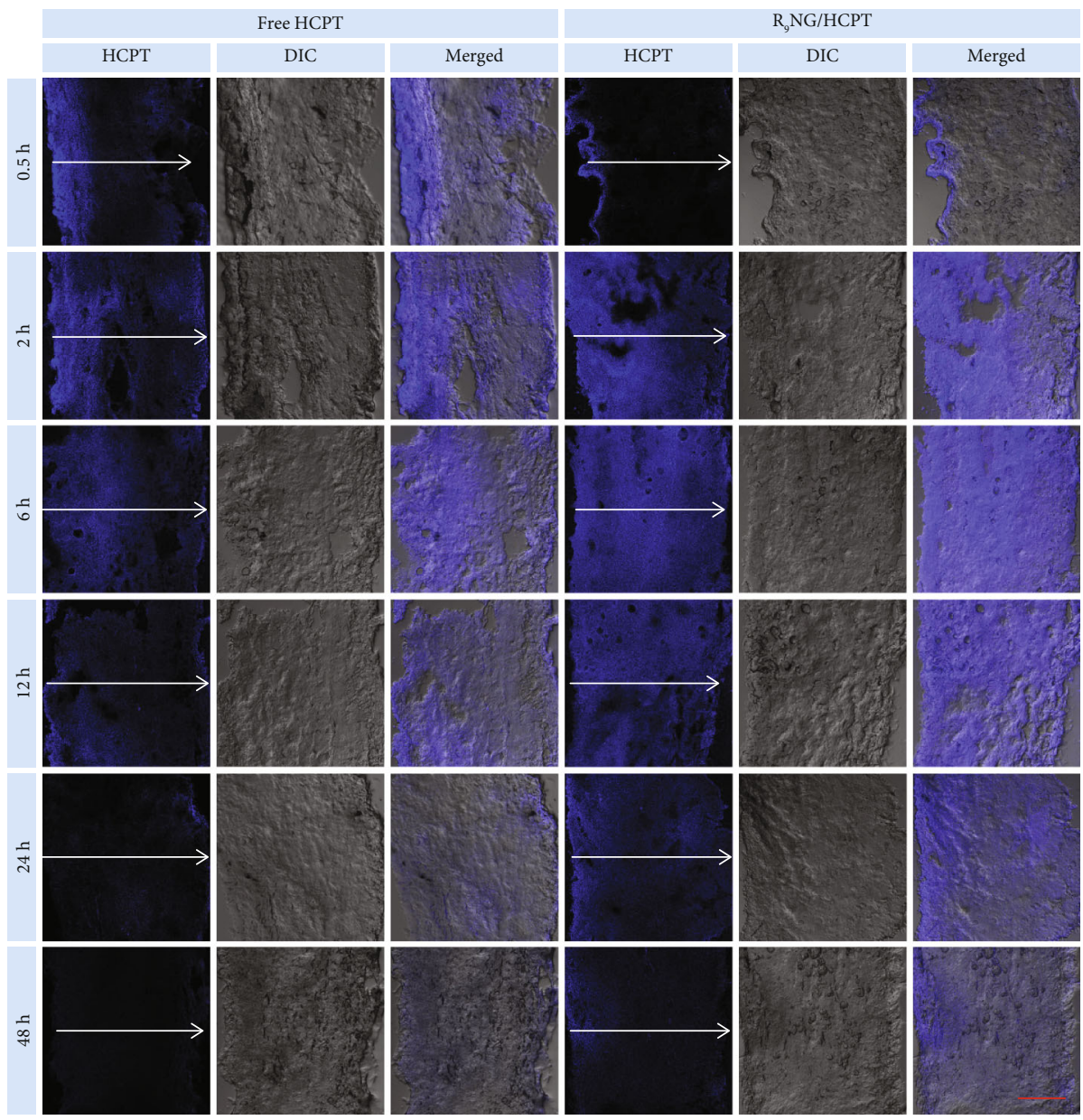

(a)
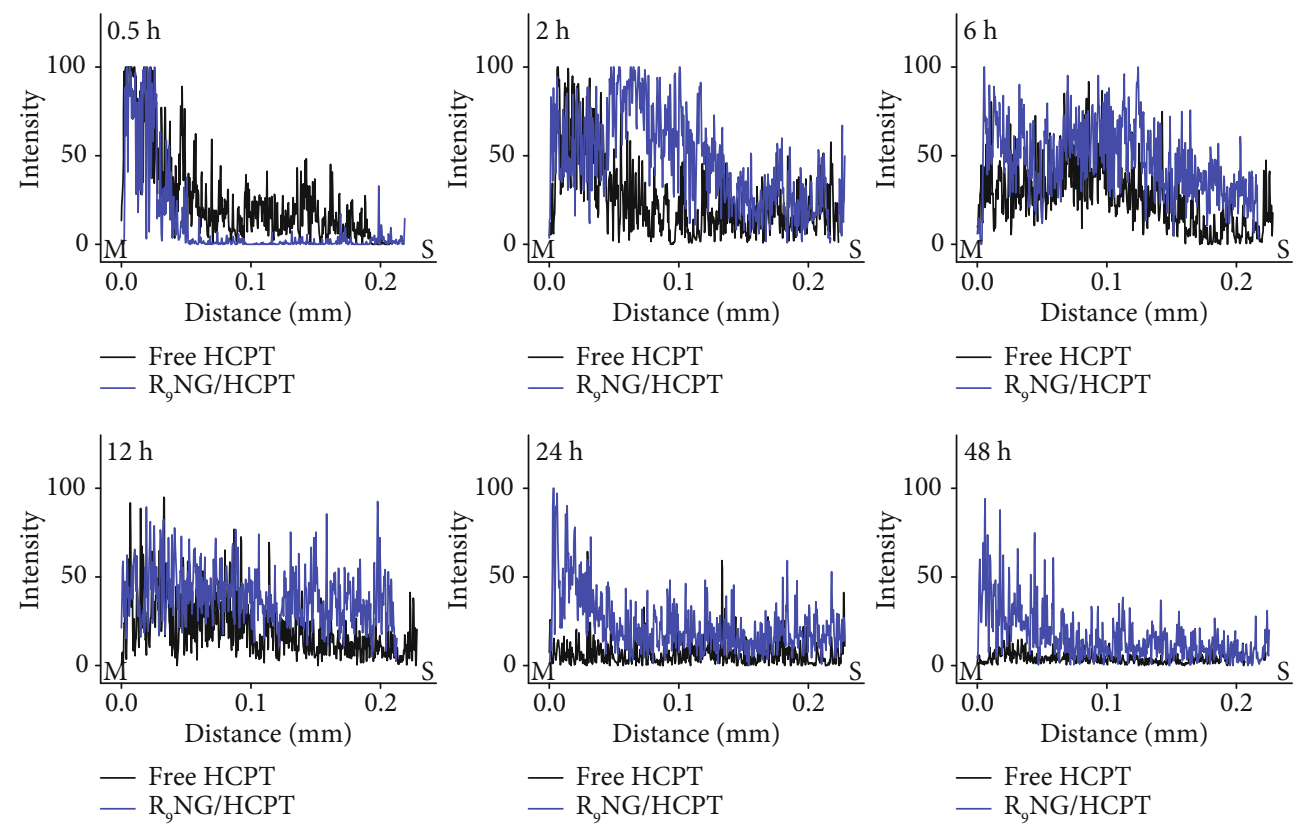

- Free HCPT

- $\mathrm{R}_{9} \mathrm{NG} / \mathrm{HCPT}$

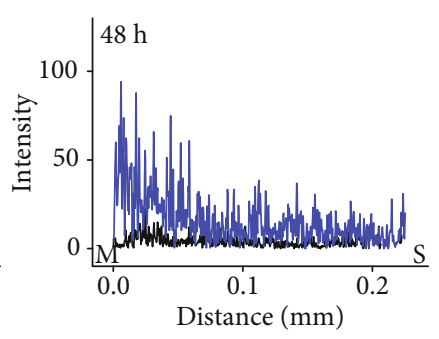

— Free HCPT
- R $\mathrm{R}$ NG/HCPT

(b)

Figure 4: Penetrability. (a) Penetration depth of free HCPT and $\mathrm{R}_{9}$ NG/HCPT was observed by CLSM. (b) Bladder penetration quantification of HCPT fluorescence intensity. The arrow represents the direction of HCPT penetration. The scale bar in (a) represents $100 \mu \mathrm{m}$. M, mucous membrane; $\mathrm{S}$, serous membrane. 

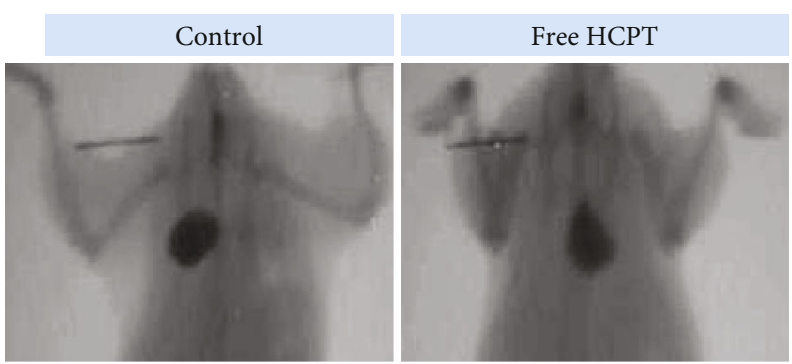

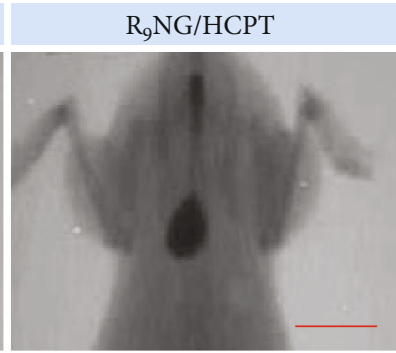

(a)
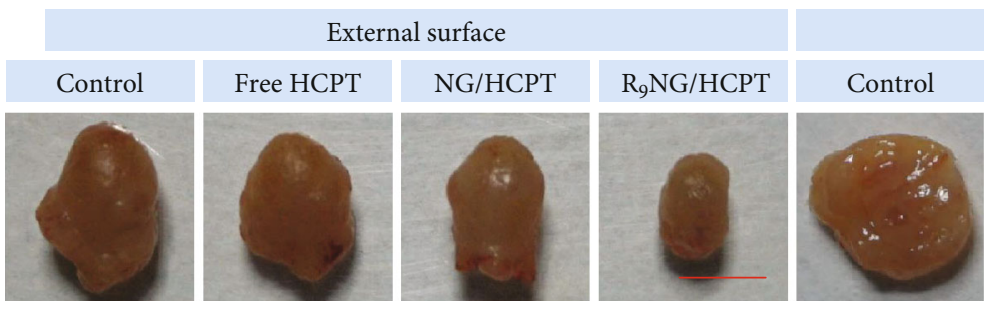

(b)

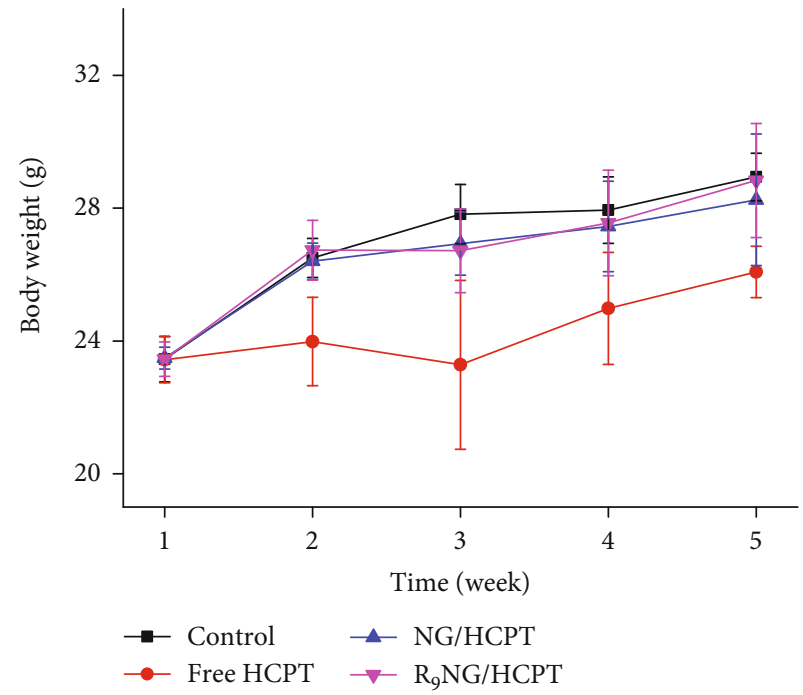

(c)

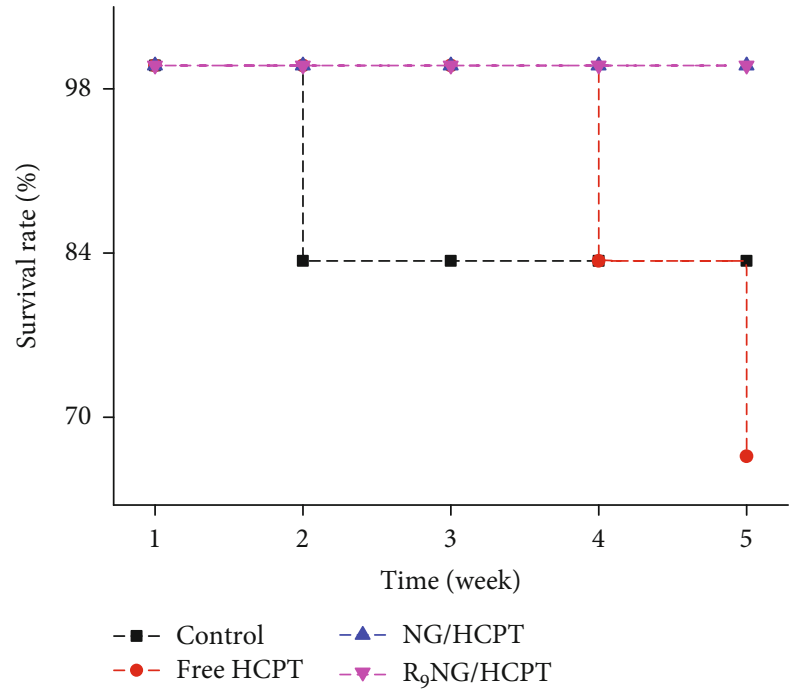

(d)

FIGURE 5: In vivo anti-tumor efficacy on orthotopic BC in C57Bl/6 mice. (a) Cystography and (b) external and internal surface of bladders after intravesical chemotherapy with PBS as a control, free HCPT, NG/HCPT, or $\mathrm{R}_{9} \mathrm{NG} / \mathrm{HCPT}$. (c) Evolution of body weight and (d) survival rate during the experiments. The scale bars represent (a) $1.0 \mathrm{~cm}$ and (b) $0.5 \mathrm{~cm}$, respectively. Data are presented as mean \pm standard deviation $(n=6)$.

of experimental animals in comparison with the control group. Intravesical instillation of $\mathrm{R}_{9} \mathrm{NG} / \mathrm{HCPT}$ has shown an especially sig-nificant survival advantage in the murine orthotopic BC model. These encouraging results benefited from the excellent biochemical properties of $\mathrm{R}_{9} \mathrm{NG} / \mathrm{HCPT}$. $\mathrm{R}_{9} \mathrm{NG} / \mathrm{HCPT}$ possessed improved mucoadhesiveness and permea-bility and could selectively release HCPT within cancer cells. All these results suggested that the tumor growth inhibition effect was enhanced, while the systemic toxicity was decreased, which confirmed the reliable biological safety of $\mathrm{R}_{9} \mathrm{NG} / \mathrm{HCPT}$.

To further accurately evaluate the anti-tumor activity of $\mathrm{R}_{9} \mathrm{NG} / \mathrm{HCPT}$, the histopathological examination of the bladders was performed after the completion of intravesical chemotherapy. For hematoxylin and eosin (H\&E) staining (Figure 6(a) and Figure S8A, Supporting Information), large amounts of cancer cells with spherical or spindle nuclei and prominent atypia were observed in the bladder section of the PBS group. Fortunately, the tumor cells showed karyopyknosis, chromatic agglutination, apoptotic body, and ill-defined morphology in the bladder administrated with $\mathrm{R}_{9} \mathrm{NG} / \mathrm{HCPT}$, indicating the predominant contribution of $\mathrm{R}_{9} \mathrm{NG} / \mathrm{HCPT}$ in tumor suppression.

Moreover, the detection of apoptotic DNA fragments was performed using the terminal deoxyribonucleotide transferase (TdT)-mediated biotin-16-dUTP nick-end labeling 


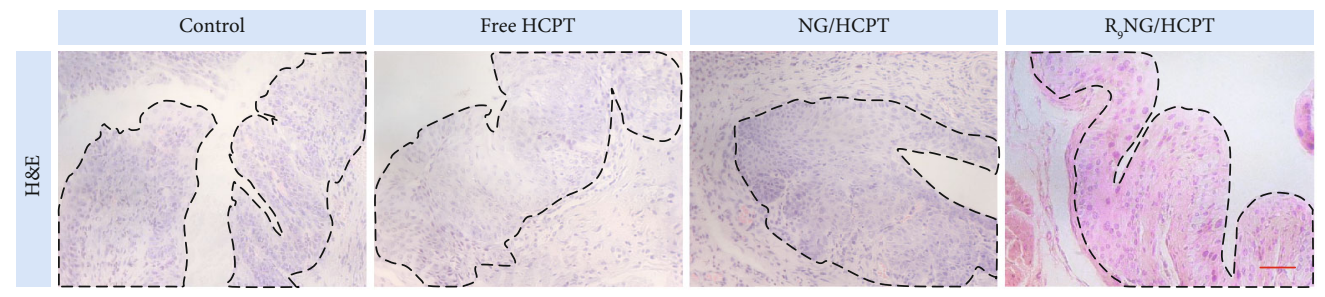

(a)

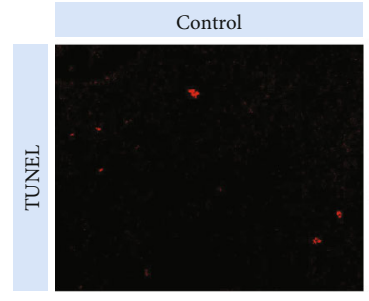

Free HCPT

NG/HCPT

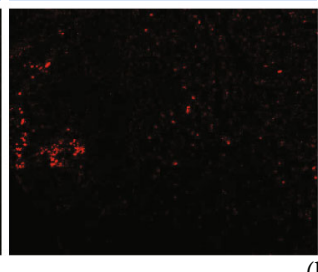

(b)

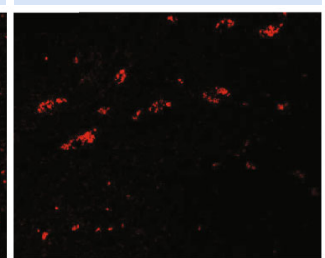

DAPI
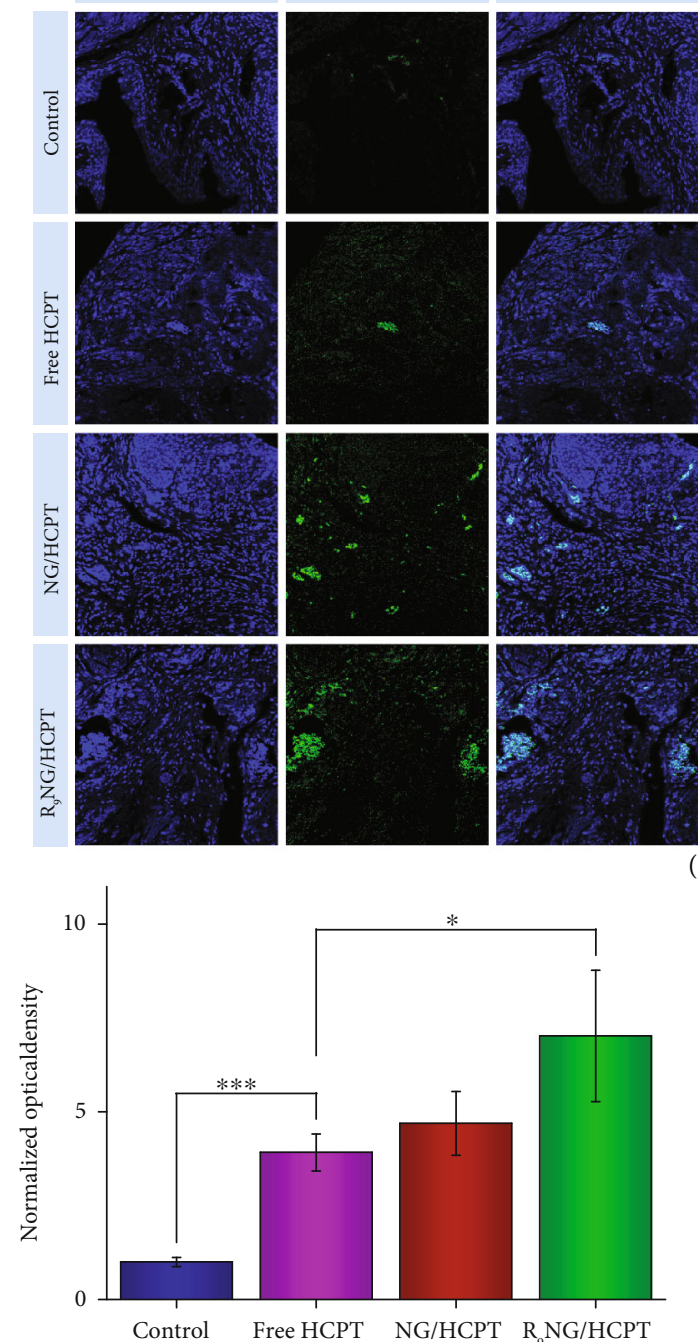

(d)
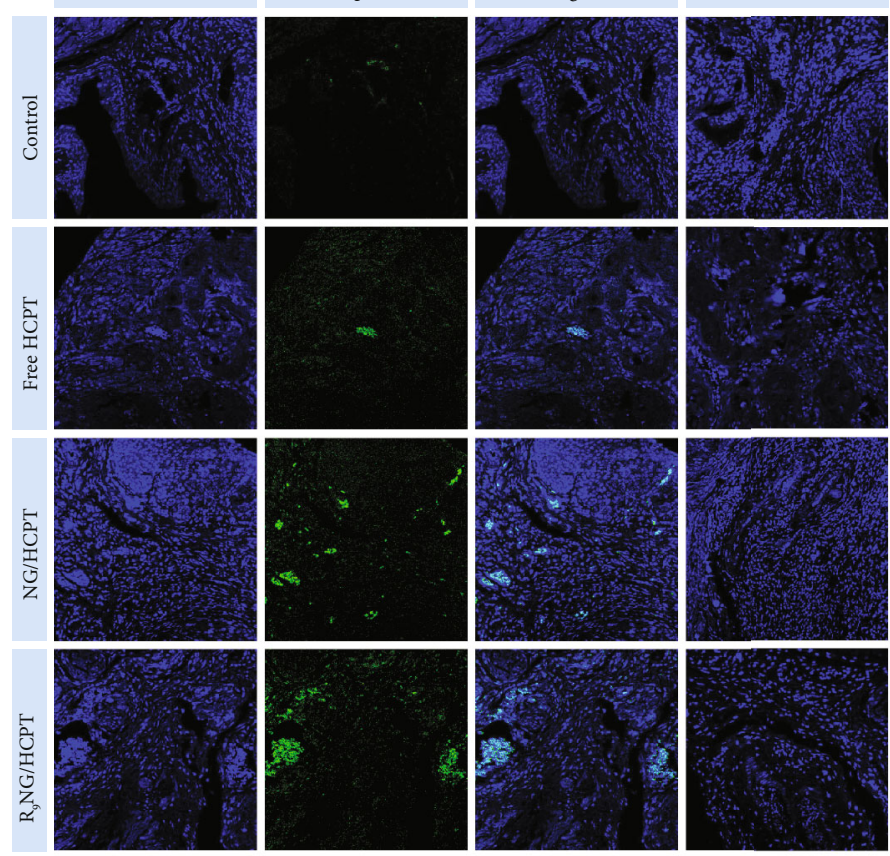

(c)
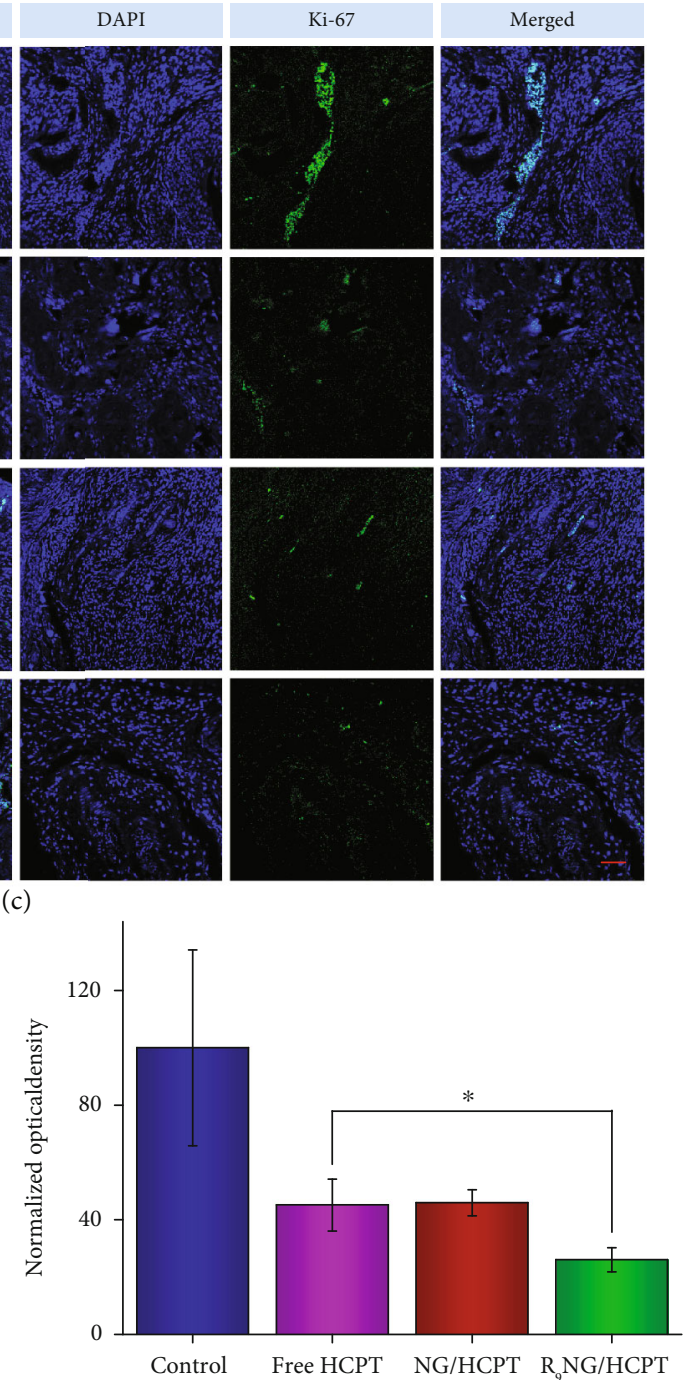

(e)

FIgURE 6: Histopathology and immunofluorescence of orthotopic BC in C57Bl/6 mice. (a) Histopathological (i.e., H\&E), (b) apoptosis (i.e., TUNEL), and (c) immunofluorescence (i.e., caspase-3 and Ki-67) analysis of tumor tissue sections after treatment with PBS as a control, free HCPT, NG/HCPT, or $\mathrm{R}_{9} \mathrm{NG} / \mathrm{HCPT}$. The scale bars in (a), (b), and (c) represent $50 \mu \mathrm{m}$. The quantitative analysis of (d) caspase-3 and (e) Ki-67 expression after treatments with different HCPT formulations. Data are presented as mean \pm standard deviation $\left(n=3 ;{ }^{*} P<0.05,{ }^{* * *} P<0.001\right)$. 
(TUNEL) method. As depicted in Figure 6(b), the red fluorescence of $\mathrm{Cy} 3$ was observed in all the bladder sections. In particular, the bladder treated with $\mathrm{R}_{9} \mathrm{NG} / \mathrm{HCPT}$ showed a larger area of flake-like red fluorescence as compared to those of the other three groups.

Caspase-3, a crucial mediator of programmed cell apoptosis, is located downstream of apoptotic response [34]. To further analyze the apoptosis mediated by $\mathrm{R}_{9} \mathrm{NG} / \mathrm{HCPT}$, immunofluorescence staining for caspase-3 was performed. The expression of caspase- 3 in mouse and rat bladder tissues was depicted in Figure 6(c) and Figure S8B, Supporting Information, respectively. The level of caspase- 3 was much higher in the section administrated with $\mathrm{R}_{9} \mathrm{NG} / \mathrm{HCPT}$ as compared to that administrated with free HCPT or NG/ HCPT. A feeble positive signal was observed in the bladder section of control group. The phenomenon indicated that a large number of cells were undergoing apoptosis in the $\mathrm{R}_{9} \mathrm{NG} / \mathrm{HCPT}$ group.

$\mathrm{Ki}-67$, a cell proliferative marker, is expressed from prophase to anaphase [35]. To confirm the cell proliferation status after treatment with $\mathrm{R}_{9} \mathrm{NG} / \mathrm{HCPT}$, the expression of Ki-67 in different experimental animals was monitored by immunofluorescence, as shown in Figure 6(c) and Figure S8B, Supporting Information. The strongest positive signal was recorded in the control group. The proportion of Ki-67-positive cells diminished significantly due to the use of different HCPT formulations, especially $\mathrm{R}_{9} \mathrm{NG} / \mathrm{HCPT}$, which demonstrated that the growth of $\mathrm{BC}$ cells was obviously suppressed by $\mathrm{R}_{9} \mathrm{NG} / \mathrm{HCPT}$.

To further confirm the expression of caspase- 3 and $\mathrm{Ki}$ 67 , the quantitative analysis was performed by a similar method as mucoadhesiveness mentioned above. The quantitative data described the order of caspase- 3 expression as $\mathrm{R}_{9} \mathrm{NG} / \mathrm{HCPT}>\mathrm{NG} / \mathrm{HCPT}>$ free HCPT $>$ PBS as a control. More importantly, the expression of caspase-3 in the $\mathrm{R}_{9} \mathrm{NG} / \mathrm{HCPT}$ group was 7.0 (Figure $6(\mathrm{~d}), \mathrm{BC}$ in mouse) and 5.6 (Figure S8C, BC in rat) times higher as compared to that of the free HCPT group, which demonstrated that a large number of cells were undergoing apoptosis in the $\mathrm{R}_{9} \mathrm{NG}$ / HCPT group. The quantitative data of Ki-67 demonstrated that the Ki-67 signal was sorted as PBS as a control > free $\mathrm{HCPT}>\mathrm{NG} / \mathrm{HCPT}>\mathrm{R}_{9} \mathrm{NG} / \mathrm{HCPT}$, demonstrating a signifi-cant proliferation inhibition of tumor cells induced by $\mathrm{R}_{9} \mathrm{NG} / \mathrm{HCPT}$ (Figure 6(e) and Figure S8D, Supporting Information). All these results indicated that $\mathrm{R}_{9} \mathrm{NG} / \mathrm{HCPT}$ possessed unique advantages for BC chemotherapy.

\section{Conclusion}

In summary, a cationic $\mathrm{R}_{9}$-decorated disulfide-crosslinked $\mathrm{R}_{9} \mathrm{NG} / \mathrm{HCPT}$ with enhanced mucoadhesion and penetration toward the bladder mucosa was designed and developed for intravesical chemotherapy. PEG and $\mathrm{R}_{9}$ endowed $\mathrm{R}_{9} \mathrm{NG} /$ HCPT with upregulated mucoadhesiveness by the nonspecific and electrostatic interactions, respectively, and $R_{9}$ further promoted the permeability of laden nanogel within the bladder wall. The advanced properties of $\mathrm{R}_{9} \mathrm{NG} / \mathrm{HCPT}$ overcame the disadvantages of clinical chemotherapeutic drugs, which will bring unpredictable benefits to BC patients and increase overall survival. More importantly, the reductionresponsive property enabled $\mathrm{R}_{9} \mathrm{NG} / \mathrm{HCPT}$ to release the cargo specifically in cancer cells triggered by the intracellular reductive microenvironment. This means that $\mathrm{R}_{9} \mathrm{NG} / \mathrm{HCPT}$ may achieve the same intracellular drug concentration as clinical chemotherapeutic agents, but the administered quantity of $\mathrm{R}_{9} \mathrm{NG} / \mathrm{HCPT}$ will decrease. The property avoided the potential risk of drug resistance due to high-dose chemotherapy, which was fundamentally critical for patients with BC. The extremely outstanding anti-tumor efficacy and the negligible systemic toxicity pave the way of $\mathrm{R}_{9} \mathrm{NG} / \mathrm{HCPT}$ for further progress in the clinical applications.

\section{Data Availability}

All data are available in the manuscript and supplementary materials, or from the authors.

\section{Conflicts of Interest}

The authors declare no conflict of interest.

\section{Acknowledgments}

The study was financially supported by the National Natural Science Foundation of China (Grant Nos. 51973216, 51873207, 51833010, and 51803006), the Science and Technology Development Program of Jilin Province (Grant No. 20200404182YY), and the Youth Innovation Promotion Association of Chinese Academy of Sciences (Grant No. 2019005).

\section{Supplementary Materials}

Supplementary 1. Materials and Methods. Supplementary 2. Figure S1: ${ }^{1} \mathrm{H}$ NMR spectrum of aPEG-P(LP-co-LC) in deuterated trifluoroacetic acid (TFA- $d)$. Supplementary 3. Figure S2: ${ }^{1} \mathrm{H}$ NMR spectrum of $t$-Boc-NH-PEG-P(LP-co-LC) in deuterated trifluoroacetic acid (TFA-d). Supplementary 4. Figure S3: ${ }^{1} \mathrm{H}$ NMR spectrum of $\mathrm{NH}_{2}$-PEG-P(LP-co-LC) in deuterated trifluoroacetic acid (TFA-d). Supplementary 5. Figure S4: ${ }^{1} \mathrm{H}$ NMR spectra of (A) $\mathrm{R}_{9}$-PEG-P(LP-co-LC), (B) Mal-PEG-P(LP-co-LC), and (C) $\mathrm{R}_{9} \mathrm{C}$ in deuterated trifluoroacetic acid (TFA-d). Supplementary 6. Figure S5: FCM analysis calculated apoptotic cell populations after coincubating human BC 5637 cells with PBS as a control, free HCPT, NG/HCPT, or $\mathrm{R}_{9} \mathrm{NG} / \mathrm{HCPT}$ for $48 \mathrm{~h}$. The lower-left (Q3), lower-right (Q4), upper-right (Q1), and upper-left (Q2) quadrants in each panel indicated the populations of healthy, early and late apoptotic, and necrotic cells, respectively. Supplementary 7. Figure S6: In vivo biodistribution of free HCPT and $\mathrm{R}_{9} \mathrm{NG} / \mathrm{HCPT}$. Data are presented as mean \pm standard deviation $\left(n=3 ;{ }^{* *} P<0.01\right)$. Supplementary 8. Figure S7: In vivo anti-tumor efficacy on orthotopic BC in SD rats. (A) Cystography and (B) external and internal surface of bladders after intravesical chemotherapy with PBS as a control, free HCPT, or $\mathrm{R}_{9} \mathrm{NG} / \mathrm{HCPT}$. (C) Evolution of body weight and (D) survival rate during the experiments. The scale bars represent (A) $1.0 \mathrm{~cm}$ and (B) $0.5 \mathrm{~cm}$, respectively. Data are presented as mean \pm standard deviation $(n=8)$. Supplementary 9. Figure S8: histopathology and 
immunofluorescence of orthotopic BC in SD rats. (A) Histopathological (i.e., $\mathrm{H} \& \mathrm{E}$ ) and (B) immunofluorescence (i.e., caspase-3 and Ki-67) analysis of tumor tissue sections after treatment with PBS as a control, free HCPT, or $\mathrm{R}_{9} \mathrm{NG} / \mathrm{HCPT}$. The scale bars in (A) and (B) represent $0.5 \mathrm{~cm}$ and $100 \mu \mathrm{m}$, respectively. The quantitative analysis of (C) caspase- 3 and (D) Ki-67 expression after treatments with different HCPT formulations. Data are presented as mean \pm standard deviation $\left(n=3 ;{ }^{*} P<0.05,{ }^{* * *} P<0.001\right)$. (Supplementary Materials)

\section{References}

[1] A. M. Kamat, M. Colombel, D. Sundi et al., "BCG-unresponsive non-muscle-invasive bladder cancer: Recommendations from the IBCG," Nature Reviews Urology, vol. 14, no. 4, pp. 244-255, 2017.

[2] W. S. Tan, A. Panchal, L. Buckley et al., "Radiofrequencyinduced thermo-chemotherapy effect versus a second course of bacillus Calmette-Guérin or institutional standard in patients with recurrence of non-muscle-invasive bladder cancer following induction or maintenance bacillus Calmette-Guérin therapy (HYMN): A phase III, open-label, randomised controlled trial," European Urology, vol. 75, no. 1, pp. 63-71, 2019.

[3] J. D. Kelly, W. S. Tan, N. Porta et al., "BOXIT—A randomised phase III placebo-controlled trial evaluating the addition of celecoxib to standard treatment of transitional cell carcinoma of the bladder (CRUK/07/004)," European Urology, vol. 75, no. 4, pp. 593-601, 2019.

[4] J. Hedegaard, P. Lamy, I. Nordentoft et al., "Comprehensive transcriptional analysis of early-stage urothelial carcinoma," Cancer Cell, vol. 30, no. 1, pp. 27-42, 2016.

[5] C. Berdik, "Unlocking bladder cancer," Nature, vol. 551, no. 7679, pp. S34-S35, 2017.

[6] R. M. Davis, B. Kiss, D. R. Trivedi, T. J. Metzner, J. C. Liao, and S. S. Gambhir, "Surface-enhanced Raman scattering nanoparticles for multiplexed imaging of bladder cancer tissue permeability and molecular phenotype," ACS Nano, vol. 12, no. 10, pp. 9669-9679, 2018.

[7] C. M. Caramella, S. Rossi, F. Ferrari, M. C. Bonferoni, and G. Sandri, "Mucoadhesive and thermogelling systems for vaginal drug delivery," Advanced Drug Delivery Reviews, vol. 92, pp. 39-52, 2015.

[8] M. R. Kang, G. Yang, R. F. Place et al., "Intravesical delivery of small activating RNA formulated into lipid nanoparticles inhibits orthotopic bladder tumor growth," Cancer Research, vol. 72, no. 19, pp. 5069-5079, 2012.

[9] Y. Y. Wang, S. K. Lai, J. S. Suk, A. Pace, R. Cone, and J. Hanes, "Addressing the PEG mucoadhesivity paradox to engineer nanoparticles that "slip" through the human mucus barrier," Angewandte Chemie, International Edition, vol. 47, no. 50, pp. 9726-9729, 2008.

[10] S. Wieczorek, A. Dallmann, Z. Kochovski, and H. G. Borner, "Advancing drug formulation additives toward precision additives with release mediating peptide interlayer," Journal of the American Chemical Society, vol. 138, no. 30, pp. 9349-9352, 2016.

[11] J. Hao, G. Song, T. Liu et al., "In vivo long-term biodistribution, excretion, and toxicology of PEGylated transition-metal dichalcogenides $\mathrm{MS}_{2}(\mathrm{M}=\mathrm{Mo}, \mathrm{W}, \mathrm{Ti})$ nanosheets," Advanced Science, vol. 4, no. 1, article 1600160, 2017.
[12] C. Douat, C. Aisenbrey, S. Antunes et al., "A cell-penetrating foldamer with a bioreducible linkage for intracellular delivery of DNA," Angewandte Chemie, International Edition, vol. 54, no. 38, pp. 11133-11137, 2015.

[13] B. P. Meloni, D. Milani, A. B. Edwards et al., "Neuroprotective peptides fused to arginine-rich cell penetrating peptides: Neuroprotective mechanism likely mediated by peptide endocytic properties," Pharmacology and Therapeutics, vol. 153, pp. 36-54, 2015.

[14] N. Q. Shi and X. R. Qi, "Taming the wildness of "TrojanHorse" peptides by charge-guided masking and proteasetriggered demasking for the controlled delivery of antitumor agents," ACS Applied Materials \& Interfaces, vol. 9, no. 12, pp. 10519-10529, 2017.

[15] M. Li, S. Schlesiger, S. K. Knauer, and C. Schmuck, “A tailormade specific anion-binding motif in the side chain transforms a tetrapeptide into an efficient vector for gene delivery," Angewandte Chemie, International Edition, vol. 54, no. 10, pp. 2941-2944, 2015.

[16] N. Zhang, Z. Yan, X. Zhao, Q. Chen, and M. Ma, "Efficient mini-transporter for cytosolic protein delivery," ACS Applied Materials \& Interfaces, vol. 8, no. 39, pp. 25725-25732, 2016.

[17] A. A. Hakimi, E. Reznik, C. H. Lee et al., "An integrated metabolic atlas of clear cell renal cell carcinoma," Cancer Cell, vol. 29, no. 1, pp. 104-116, 2016.

[18] H. Guo, W. Xu, J. Chen et al., "Positively charged polypeptide nanogel enhances mucoadhesion and penetrability of 10 hydroxycamptothecin in orthotopic bladder carcinoma," Journal of Controlled Release, vol. 259, pp. 136-148, 2017.

[19] D. A. Zaharoff, B. S. Hoffman, H. B. Hooper et al., "Intravesical immunotherapy of superficial bladder cancer with chitosan/interleukin-12," Cancer Research, vol. 69, no. 15, pp. 61926199, 2009.

[20] P. M. K. Westcott, K. D. Halliwill, M. D. To et al., "The mutational landscapes of genetic and chemical models of KRASdriven lung cancer," Nature, vol. 517, no. 7535, pp. 489-492, 2015.

[21] G. Aldrian, A. Vaissière, K. Konate et al., "PEGylation rate influences peptide-based nanoparticles mediated siRNA delivery in vitro and in vivo," Journal of Controlled Release, vol. 256, pp. 79-91, 2017.

[22] X. R. Feng, J. X. Ding, R. Gref, and X. S. Chen, "Poly $(\beta-$ cyclodextrin)-mediated polylactide-cholesterol stereocomplex micelles for controlled drug delivery," Chinese Journal of Polymer Science, vol. 35, no. 6, article 1932, pp. 693699, 2017.

[23] J. Chen, J. Ding, W. Xu et al., "Receptor and microenvironment dual-recognizable nanogel for targeted chemotherapy of highly metastatic malignancy," Nano Letters, vol. 17, no. 7, pp. 4526-4533, 2017.

[24] J. F. Zhang, H. L. Xiong, J. L. Cao et al., "A cell-penetrating whole molecule antibody targeting intracellular $\mathrm{HBx}$ suppresses hepatitis B virus via TRIM21-dependent pathway," Theranostics, vol. 8, no. 2, pp. 549-562, 2018.

[25] T. Y. Lin, Y. Li, Q. Liu et al., "Novel theranostic nanoporphyrins for photodynamic diagnosis and trimodal therapy for bladder cancer," Biomaterials, vol. 104, pp. 339-351, 2016.

[26] W. Wei, Z. G. Yue, J. B. Qu, H. Yue, Z. G. Su, and G. H. Ma, "Galactosylated nanocrystallites of insoluble anticancer drug 
for liver-targeting therapy: An in vitro evaluation," Nanomedicine, vol. 5, no. 4, pp. 589-596, 2010.

[27] B. S. Lele and A. S. Hoffman, "Mucoadhesive drug carriers based on complexes of poly(acrylic acid) and PEGylated drugs having hydrolysable PEG-anhydride-drug linkages," Journal of Controlled Release, vol. 69, no. 2, pp. 237-248, 2000.

[28] Y. Lee, T. E. Deelman, K. Chen, D. S. Y. Lin, A. Tavakkoli, and J. M. Karp, "Therapeutic luminal coating of the intestine," Nature Materials, vol. 17, no. 9, pp. 834-842, 2018.

[29] J. Chen, J. Ding, Y. Wang et al., "Sequentially responsive shell-stacked nanoparticles for deep penetration into solid tumors," Advanced Materials, vol. 29, no. 32, article 1701170, 2017.

[30] S. Futaki and I. Nakase, "Cell-surface interactions on argininerich cell-penetrating peptides allow for multiplex modes of internalization," Accounts of Chemical Research, vol. 50, no. 10, pp. 2449-2456, 2017.

[31] A. J. Vandeveer, J. K. Fallon, R. Tighe, H. Sabzevari, J. Schlom, and J. W. Greiner, "Systemic immunotherapy of non-muscle invasive mouse bladder cancer with avelumab, an anti-PDL1 immune checkpoint inhibitor," Cancer Immunology Research, vol. 4, no. 5, pp. 452-462, 2016.

[32] H. Guo, F. Li, W. Xu et al., "Mucoadhesive cationic polypeptide nanogel with enhanced penetration for efficient intravesical chemotherapy of bladder cancer," Advanced Science, vol. 5, no. 6, article 1800004, 2018.

[33] R. Kinoshita, Y. Ishima, V. T. G. Chuang et al., "Improved anticancer effects of albumin-bound paclitaxel nanoparticle via augmentation of EPR effect and albumin-protein interactions using $S$-nitrosated human serum albumin dimer," Biomaterials, vol. 140, pp. 162-169, 2017.

[34] N. Ma, P. Liu, N. He, N. Gu, F. G. Wu, and Z. Chen, "Action of gold nanospikes-based nanoradiosensitizers: Cellular internalization, radiotherapy, and autophagy," ACS Applied Materials \& Interfaces, vol. 9, no. 37, pp. 31526-31542, 2017.

[35] S. Cuylen, C. Blaukopf, A. Z. Politi et al., "Ki-67 acts as a biological surfactant to disperse mitotic chromosomes," Nature, vol. 535, no. 7611, pp. 308-312, 2016. 